\title{
New observations from the SWIM radar on board CFOSAT: instrument validation and ocean wave measurement assessment
}

\author{
D. Hauser ${ }^{(1)}$ Member, IEEE, C. Tourain ${ }^{(2)}$, L. Hermozo $^{(2)}$, D. Alraddawi ${ }^{(1)}$ L. Aouf $^{(3)}$, B. \\ Chapron $^{(4)}$, A. Dalphinet ${ }^{(3)}$, L. Delaye ${ }^{(5)}$, M. Dalila ${ }^{(6)}$, E. Dormy $^{(7)}$, F. Gouillon ${ }^{(2)}$, V.

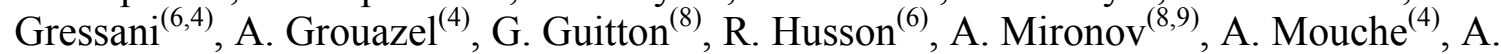 \\ Ollivier $^{(6)}$, L. Oruba ${ }^{(1)}$, F. Piras ${ }^{(6)}$, R. Rodriguez Suquet ${ }^{(2)}$, P. Schippers ${ }^{(5)}$, C. Tison ${ }^{(2)}$, Ngan \\ $\operatorname{Tran}^{(6)}$
}

\begin{abstract}
(C) 2020 IEEE. Personal use of this material is permitted. Permission from IEEE must be obtained for all other uses, in any current or future media, including reprinting/republishing this material for advertising or promotional purposes, creating new collective works, for resale or redistribution to servers or lists, or reuse of any copyrighted component of this work in other works.
\end{abstract}

\begin{abstract}
This paper describes first results obtained from the SWIM (Surface Waves Investigation and Monitoring) instrument carried by CFOSAT (China France Oceanography Satellite), which was launched on October $29^{\text {th }}, 2018$. SWIM is a Ku-Band radar with a near-nadir scanning beam geometry. It was designed to measure the spectral properties of surface ocean waves. First, the good behavior of the instrument is illustrated. It is then shown that the nadir products (significant wave height, normalized radar cross-section and wind speed) exhibit an accuracy similar to standard altimeter missions, thanks to a new retracking algorithm, which compensates a lower sampling rate compared to standard altimetry missions. The off-nadir beam observations are analyzed in details. The normalized radar cross-section varies with incidence and wind speed as expected from previous studies presented in the literature. We illustrate that, in order to retrieve the wave spectra from the radar backscattering fluctuations, it is crucial to apply a speckle correction derived from the observations. Directional spectra of ocean waves and their mean parameters are then compared to wave model data at the global scale and to in situ data from a selection of case studies. The good efficiency of SWIM to provide the spectral properties of ocean waves in the wavelength range $[70 \mathrm{~m}-500 \mathrm{~m}]$ is illustrated. The main limitations are discussed, and the perspectives to improve data quality are presented.
\end{abstract}

Index Terms-Radar measurements, spaceborne radar, sea surface, scatterometer, altimeter, speckle noise, ocean wave spectra.

\section{INTRODUCTION}

Since October 2018, a new space-borne system for measuring ocean surface parameters has been deployed, namely the CFOSAT (China France Oceanography Satellite) developed under the responsibilities of the French and Chinese Space agencies (The Centre National d'Etudes Spatiales CNES, The Chinese National Space Agency CNSA and the National Satellite Oceanic Application Service NSOAS). This satellite was designed to monitor, at the global scale, ocean surface winds and waves so as to improve wind and wave forecast for marine meteorology (including severe events), ocean dynamics modeling and prediction, climate variability knowledge, fundamental knowledge of surface processes, etc. CFOSAT also offers an opportunity to complement other satellite missions for the estimation of land surface parameters (in particular soil moisture and soil roughness), and polar ice sheet characteristics. The main objectives and characteristics were already presented in [1] and [2].

This mission is a very innovative one, for several reasons. First, thanks to its near-nadir scatterometer SWIM (Surface Investigation and Monitoring), it is the first time that directional spectra of ocean waves are produced systematically with a realaperture scanning radar system. With this concept, the backscattered signal is sampled with a high horizontal resolution of only a few meters in the range direction but averaged over a large footprint (several kilometers) in the perpendicular direction. Hence, waves with a significant component in the azimuth direction are averaged over many wavelengths and therefore contribute little to the radar signal. Only long waves travelling in the range direction will be seen because of the sensitivity of the radar backscatter to the local tilting of the surface. Their direction is determined from the known pointing direction of the antenna and from its rotating capability insuring a $360^{\circ}$ detection. This concept is a useful complement to SAR (Synthetic Aperture Radar) observations which are also used to provide wave spectra over the ocean, but with frequent smearing effects, for waves shorter than about $200 \mathrm{~m}$ propagating with a component in the along-track directions [3], even if recent results by [4] show that it is possible to extract consistent information on wind waves from waves propagation across-track. Secondly, the mission provides simultaneous and collocated measurements of wind and waves, which is very important to offer a better validation of wave models, progress in the analysis of wave physics and air-sea interaction processes. Furthermore, the two instruments 
on-board CFOSAT (SWIM and a wind scatterometer called SCAT) are new technical concepts (with for both, a beam rotating scanning geometry) which have pushed the technology ahead.

In this context, the aim of this paper is to discuss the first results obtained with the SWIM instrument from the verification phase. The results presented here summarize the results obtained by the Wind and Wave Experts groups organized by CNES to validate the SWIM data.

The manuscript is organized as follows: section II recalls the main characteristics of the mission and of the SWIM instrument. Section III summarizes the main content of the scientific products and explains the methods and tools used for the validation. Section IV provides a summary on the SWIM instrument performance. Section V shows results on the nadir beam products (wave height, normalized radar cross-section and wind speed similarly to other altimeter missions). Section VI discusses the results obtained on the normalized radar crosssection in a diversity of observation geometry (diversity of near-nadir incidence and azimuth angles). Section VII presents results of the fluctuation and speckle spectra which are intermediate quantities between the normalized radar crosssection and the final Level 2 product (wave spectra). Section VIII presents an assessment of the wave spectral parameters. Finally, section IX summarizes the results, indicates the work under progress to improve the processing and the products, and concludes.

\section{MAIN CHARACTERISTICS OF THE MISSION AND OF THE SWIM INSTRUMENT}

\section{A. Mission}

To meet the objectives recalled in the introduction, the satellite carries two payloads; both are Ku-band radars scanning around the vertical axis:

- the wave scatterometer SWIM operating at $13.575 \mathrm{GHz}$, a rotating 6-beam radar at small incidence angles $\left(0\right.$ to $\left.10^{\circ}\right)$ [2],

- the wind scatterometer SCAT operating at $13.256 \mathrm{GHz}$, a fan-beam radar at larger incidence angles (26 to $46^{\circ}$ ) [5].

CFOSAT was launched on October the $29^{\text {th }}, 2018$ on a low altitude sun-synchronous orbit (around $500 \mathrm{~km}$ ). The main characteristics of the CFOSAT orbit are summarized in Table 1.

The main objective of SWIM is to provide directional wave spectra as explained in [2]. The main products delivered to users are:

- Significant wave height (Hs) and wind speed from nadir measurements.

- Directional wave spectra and their parameters from offnadir beams pointing at $6^{\circ}, 8^{\circ}, 10^{\circ}$ mean incidences.

- Normalized radar cross-section profiles from $0^{\circ}$ to $10^{\circ}$.

\section{B. Measurement concept and main characteristics of SWIM}

The concept of SWIM is based on a scanning-beam realaperture radar, following the ideas proposed in the 1980's by
[6,7], both for airborne and space-borne configurations. This concept has been implemented and validated on various airborne systems $[8,9,10,11]$ ) but SWIM is the first spaceborne instrument working with this concept. As recalled in [2], the main idea is that at near-nadir incidence (around $8^{\circ}-10^{\circ}$ from nadir), the normalized radar cross-section is sensitive to the local slope of the sea surface, but almost insensitive to small scale roughness effects produced by the wind, and to hydrodynamic modulations resulting from interactions between short and long waves. Hence the main factor which modulates the normalized radar cross-section is the tilt of the long-waves when the radar looks in their direction of propagation. Details on the theoretical basis of the concept and wave spectra inversion in the configuration of SWIM can be found in [2].

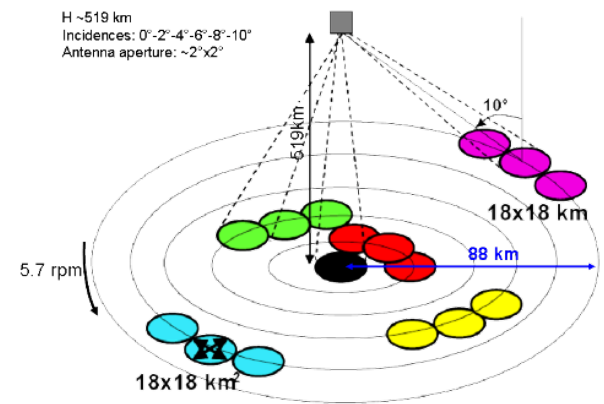

Figure 1 SWIM beam rotation and incidence angles

\begin{tabular}{|l|l|}
\hline Orbit radius & $6891.987 \mathrm{~km}$ \\
\hline Altitude at the equator & $514 \mathrm{~km}$ \\
\hline Inclination & $97.465^{\circ}$ \\
\hline Nodal period & $94.761 \mathrm{~min}$ \\
\hline Local time of descending node step between two & $23.756^{\circ}(\sim 2644 \mathrm{~km})$ \\
\hline $\begin{array}{l}\text { Longitudinal sanding nodes } \\
\text { ascend }\end{array}$ & $1.827^{\circ}(\sim 203.4 \mathrm{~km})$ \\
\hline $\begin{array}{l}\text { Longitudinal step between two } \\
\text { descending nodes }\end{array}$ & 13 days \\
\hline Cycle duration & 2 \\
\hline Number of sub-cycles & 197 \\
\hline Number of orbits per cycle & \multicolumn{1}{|c|}{ Table $1:$ CFOSAT orbit characteristics } \\
\hline
\end{tabular}

In order to exploit this concept, and provide complementary observations from nadir and from near-nadir measurements, the design was chosen as recalled below. SWIM is a Ku-Band radar with a multi-incidence and scanning azimuthal geometry (Figure 1). It illuminates the surface sequentially with 6 incidence angles: $0^{\circ}, 2^{\circ}, 4^{\circ}, 6^{\circ}, 8^{\circ}$ and $10^{\circ}$, each beam with a beam aperture in elevation and in azimuth of $1.5^{\circ}$ to $1.8^{\circ}$ (see [2]). Given the orbit height, the footprint dimension of each beam is about $18 \mathrm{~km} \times 18 \mathrm{~km}$, and the full swath for the outer beam $\left(10^{\circ}\right)$ is about $90 \mathrm{~km}$ in radius. In order to acquire data in all azimuth directions, the antenna beam is rotated at a speed rate of $5.6 \mathrm{rpm}$, which generates, when combined with the satellite advection, some overlap in the sampling of successive rotations (see [2]). Off-nadir beams at $6^{\circ}, 8^{\circ}, 10^{\circ}$ are also called the "spectral beams", as these beams are used for 2D wave spectra estimation and wave spectra parameters. 
The range detection is achieved by using a $320 \mathrm{MHz}$ chirp for the $50 \mu$ s pulses, providing a theoretical $0.47 \mathrm{~m}$ range resolution. All sequences of acquisition start with nadir sampling, which is used like on altimeter missions, to track the distance of the signal and adapt the receiving chain in consequence. The nominal sequence of acquisition follows the one given in Table 2 leading to a macro-cycle duration of about $220 \mathrm{~ms}$ (a macrocycle is defined as the sequence of illumination performed between starting again the nadir tracking). During a nominal macrocycle the azimuth rotates by about $7.5^{\circ}$. The onboard processing of the raw signal includes numerical range compression with a compensation for range migration during integration time (see [2]). Further steps in the on-board processing include incoherent time and range averaging over durations and number of samples which vary with the beam (see Table 2).

\begin{tabular}{|c|c|c|c|c|c|c|}
\hline Beam & 0 & 2 & 4 & 6 & 8 & 10 \\
\hline $\begin{array}{c}\text { Time duration } \\
\text { (ms) }\end{array}$ & 55.4 & 22.6 & 22.6 & 34.4 & 40.5 & 44.2 \\
\hline $\begin{array}{c}\text { Number of } \\
\text { integrated } \\
\text { echoes }\end{array}$ & 264 & 97 & 97 & 156 & 186 & 204 \\
\hline $\begin{array}{c}\text { Number of } \\
\text { averaged range } \\
\text { bins }\end{array}$ & 1 & 4 & 4 & 2 & 3 & 3 \\
\hline
\end{tabular}

Table 2: Main parameters of a nominal macrocycle (sequential illumination of beams $0^{\circ}$ to $10^{\circ}$ by increasing order) and associated real-time processing parameters

\section{Scientific product specification}

As described in [2], the specification on the scientific products were defined during the mission development as given in Table 3.

\begin{tabular}{|l|l|}
\hline \multirow{5}{*}{$\begin{array}{l}\text { From nadir } \\
\text { observations }\end{array}$} & $\begin{array}{l}\text { Significant wave height better than } 10 \% \text { or } 50 \\
\text { cm (maximum) }\end{array}$ \\
\cline { 2 - 3 } & $\begin{array}{l}\text { wind speed with accuracy of approximately } \pm 2 \\
\text { m/s or } 10 \% \text { (whichever is greatest) }\end{array}$ \\
\hline \multirow{5}{*}{$\begin{array}{l}\text { From off-nadir } \\
\text { beams }\end{array}$} & $\begin{array}{l}\text { Two-dimensional wave spectra at a scale of } 70 \\
\text { km } x \text { km, wavelengths detected from } 70 \mathrm{~m} \\
\text { to } 500 \mathrm{~m}\end{array}$ \\
\cline { 2 - 2 } & $\begin{array}{l}\text { Dominant wavelengths with an accuracy of } \\
10 \% \text { for up to three wave partitions of the } \\
\text { wave spectrum }\end{array}$ \\
\cline { 2 - 2 } & $\begin{array}{l}\text { Dominant directions with an accuracy of } 15^{\circ}, \\
\text { for up to three wave partitions (keeping } 180^{\circ} \\
\text { ambiguity in direction) }\end{array}$ \\
\cline { 2 - 2 } & $\begin{array}{l}\text { Significant wave height accuracy better than } \\
10 \%, \text { for up to three wave partitions }\end{array}$ \\
\hline \multirow{2}{*}{ From all beams } & $\begin{array}{l}\sigma_{0} \text { values as function of incidence and azimuth, } \\
\text { every } 0.5^{\circ} \text { in incidence and } 15^{\circ} \text { in azimuth }\end{array}$ \\
\hline
\end{tabular}

Table 3: Product specification

\section{DATA SETS AND VALIDATION MethodS}

\section{A. Products from the French Mission Centers}

From the raw data downloaded to the French Mission Center (CWWIC- CFOSAT Wind and Waves Instrument Center) the following products are generated by the processing chain and made accessible to users:

- L1a: normalized radar cross-section $\sigma_{0}$ for beams 0 to $10^{\circ}$ at the resolution and sampling of the raw downloaded data, with associated geolocalization;

- L1b: intermediate products for the generation of wave spectra, only over ocean and for beams $6^{\circ}, 8^{\circ}$, and $10^{\circ}$ : relative fluctuations of $\sigma_{0}$ within each footprint, associated fluctuation spectra with and without speckle corrections;

- L2:

- From the nadir beam $\left(0^{\circ}\right)$ over the ocean: significant wave height, normalized radar cross-section and surface wind speed (tentatively mean square slope) using a new retracking algorithm (see section $\mathrm{V}$ );

- From the nadir beam over ice and continent the main parameters are the normalized radar cross-section, and parameters of the echo shape (width of the leading edge, slope of the trailing edge in three domains)- see [12];

- For beams at $6^{\circ}, 8^{\circ}$, and $10^{\circ}$, over ocean only: $2 \mathrm{D}$ wave spectra provided in 12 directions from $0^{\circ}$ to $180^{\circ}$, and 32 wave numbers bins unequally spaced from $\mathrm{k}_{0}=0.0126$ to $\mathrm{k}_{\max }=0.2789 \mathrm{rad} / \mathrm{m}$ (with width $\Delta \mathrm{k}$ of each wavenumber bins following $\Delta \mathrm{k} / \mathrm{k}=10 \%$ ); each $2 \mathrm{D}$ spectrum is constructed from observations of successive overlapping antenna scans over $180^{\circ}$ (on each side of the track), and representative of wave cells (boxes) of about 70 x $90 \mathrm{~km}$;

- Main parameters (wave height, dominant direction and wavelength) of the wave spectra and of up to 3 wave partitions.

\section{B. Validation tools and methods}

It should first be noted that only data posterior to April $25^{\text {th }}$ 2019 have been used, because prior to this date, an error was remaining in the on-board processing (in the migration compensation algorithm) which induced an important filtering of the detected waves. Most of the validation has been done by gathering SWIM data sets acquired all over the global ocean (excluding however sea-ice covered regions). This is why we mainly rely in this paper on numerical model outputs as reference, to analyze the Level 2 SWIM data on a statistical point of view, in particular the wave spectra and their parameters. Section VIII however presents first comparisons with in situ observations.

For nadir wind and wave products, the reference is mainly the ECWAM wave model from ECMWF, and products from altimetry (Jason3, AkltiKa). For off-nadir wave spectral data, the reference comes from the MFWAM wave model. This latter is a third generation model based on the ECMWF version (ECWAM-IFS-38R2) but with a slightly modified parameterization [13] taken from the WW3 (Wave Watch 3) model [14]. The MFWAM wave products used here have a grid size of $10 \mathrm{~km}$ and are driven by 3-hourly analyzed winds from the IFS-ECMWF atmospheric system. The model MFWAM accounts waves/currents interactions with daily surface currents provided by the global PSY4-CMEMS ocean forecasting system. The wave spectrum is discretized in 24 directions and 30 frequencies starting from $0.035 \mathrm{~Hz}$ to $0.58 \mathrm{~Hz}(1300 \mathrm{~m}$ to 5 $\mathrm{m}$ of wavelength). The operational model MFWAM uses the 
assimilation of Hs from altimeters and directional wave spectra from Sentinel 1A and 1B. The MFWAM post-processor includes a partitioning method to split the spectrum into wind sea, primary and secondary swells (see section VIII).

For near-nadir normalized radar cross-sections, we used statistics of the Global Precipitation Mission (GPM) data as reference values [15].

\section{SWIM INSTRUMENTAL PERFORMANCE}

During the first month after launch, internal and antenna calibration sequences were performed continuously over several days. The analysis of the instrumental parameters such as the Point Target Response (PTR), the radar gain and the antenna rotation speed shows that all are comparable with the ground measurement values, very stable in time and well within the requirements (see Table 4).

The instrument performance is primarily evaluated via its PTR. A performant measurement of this parameter means a good precision of the radar measurement. After range compression, the PTR of a punctual target is expected to be a cardinal sinusoidal function with resolution at $3 \mathrm{~dB}$ inversely proportional to the signal bandwidth. This bandwidth defines the range resolution of the received signal. The internal calibration is used to evaluate the PTR function performance and thus the possible internal drifts related to the instrument through the derived PTR parameters. As shown in Fig.2, the inflight measured PTR (in blue) matches very well the theoretical impulse response (in red). One can see some differences between these curves, but they are all located at the low energy minima of the sinc shape PTR. The total integrated power from the measurements fits the theoretical value within $95 \%$. The fit between measured and theoretical PTR is higher than $98 \%$ if the integrated power is estimated over the principal lobe and the five first side lobes. Note that these latter parameters are part of the monitored PTR parameters used to assess the instrumental performance on a regular basis.

SWIM instrumental parameters are monitored daily with routine calibration sequences performed since December 2018 (three internal calibrations and one antenna calibration sequences per day). The performance of these parameters, from the launch to the end of commissioning phase, is synthesized in Table 4. This allows a reliable absolute calibration of SWIM $\sigma_{0}$, when correcting the acquired power from all instrumental contributions.

We can first highlight the good instrumental behavior of SWIM by its tracking mode from the nadir beam. Table 5 compares the tracking rate for SWIM instrument and for the ongoing altimeter Poseidon-3B on-board Jason 3, which uses a median tracker, similarly to SWIM. The comparison is shown over one cycle (13 days and 10 days, respectively), both over open ocean and globally. First, SWIM tracking mode shows similar performances to conventional altimetry over ocean, with a $99.96 \%$ and $99.98 \%$ coverage, respectively, during one cycle. The higher tracking performances of SWIM instrument are then highlighted over land, ice and sea ice. Thanks to the improved on-board signal processing, the tracking rate for SWIM is raised by more than 2 points regarding Poseidon-3B particularly over ice and land and reaches $98.48 \%$ over 13 days.
Secondly, the signal to noise ratio (SNR) was analyzed for each beam. Table 6 gives the averaged values of SNR, computed from the mid-swath radar gates over one cycle for the $6^{\circ}, 8^{\circ}$ and $10^{\circ}$ beams. SNR shows the contribution of instrumental noise (mainly thermal noise) with respect to the total measured signal. The higher the SNR is, the more accurate are the measurements and the instrumental noise is negligible in the total measured power. In the case of the SWIM instrument, mid-swath SNR values are $5 \mathrm{~dB}$ to $7 \mathrm{~dB}$ higher than expected worst cases, which confirms the good performance of the measurements and their high sensitivity to the ocean surface. This parameter is used to select the reliable swath of each of the spectral beams before the inversion of the 2D wave spectra.

To compute the SNR, thermal noise floor is estimated for each macrocycle and each beam. It is first estimated from the $2^{\circ}$ beam echo which exhibits in all conditions a very constant noise floor in the first range gates (at distances less than the altitude). The noise floor is estimated over the 60 first range gates. As the reception window width for the other beams does not allow the detection of a noise floor, the $2^{\circ}$ noise floor is then propagated to the other beams.

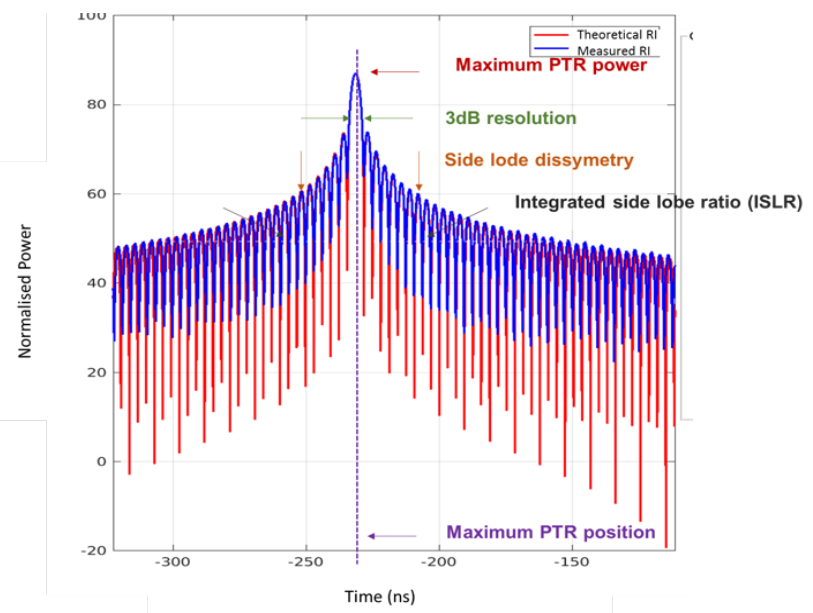

Figure 2: Point Target Response (PTR). Blue line: measured, red line: theoretical sinus cardinal function.

\begin{tabular}{|c|c|}
\hline \multicolumn{2}{|c|}{ SWIM functional and performance validation } \\
\hline Power consumption & $189 \mathrm{~W}$, stable in time \\
\hline Temperature & Compliant with spec., stable \\
\hline Coverage in tracking mode & Compliant with spec., stable \\
\hline Antenna rotation speed & Compliant with spec., stable \\
\hline Estimated pointing accuracy & $<0.1^{\circ}$ \\
\hline \multirow{4}{*}{ Point Target Response } & Maximum power variation $<0.2 \mathrm{~dB}$ \\
\cline { 2 - 2 } & Range drift $(6$ months $): 52.60 \mathrm{~mm}$ \\
\cline { 2 - 2 } & 3 dB resolution $: 2.7875 \mathrm{~ns} \pm 0.1 \mathrm{~ns}$ \\
\cline { 2 - 2 } & $\begin{array}{c}\text { Side lobe dissymmetry } \\
\left(1^{\text {st }} \text { to } 5^{\text {th }}\right)<1 \mathrm{~dB}\end{array}$ \\
\cline { 2 - 2 } & $\begin{array}{c}\text { Integrated } 1^{\text {st }} \text { side lobe ratio } \\
10.10 \mathrm{~dB} \pm 0.05 \mathrm{~dB}\end{array}$ \\
\hline Instrumental gain & Compliant with spec., stable \\
\hline \multirow{2}{*}{ Antenna gain w.r.t. azimuth } & $\begin{array}{c}\text { Differences w.r.t. on-ground } \\
\text { estimation }<0.5 \mathrm{~dB}\end{array}$ \\
\hline Table 4: SWIM functional and performances validation.
\end{tabular}

Table 4: SWIM functional and performances validation. 


\begin{tabular}{|c|c|c|}
\hline & Over ocean & Over all surfaces \\
\hline SWIM & $99.96 \%$ & $98.48 \%$ \\
\hline Poseidon-3B & $99.98 \%$ & $96.26 \%$ \\
\hline
\end{tabular}

Table 5: Tracking rate statistics for the SWIM nadir beam and for Poseidon-3B on-board Jason 3. Statistics are compared over a whole cycle (13 days for SWIM and 10 days for Jason 3) over ocean only (second column) and globally, including ocean, land, ice sheet and sea ice (third column).

\begin{tabular}{|c|c|c|c|}
\hline Beam & $6^{\circ}$ & $8^{\circ}$ & $10^{\circ}$ \\
\hline $\begin{array}{c}\text { Mean mid- } \\
\text { swath SNR }\end{array}$ & $>15 \mathrm{~dB}$ & $\sim 15 \mathrm{~dB}$ & $\sim 10 \mathrm{~dB}$ \\
\hline
\end{tabular}

Table 6: Averaged Signal to Noise Ratio computed for beams $6^{\circ}, 8^{\circ}$ and $10^{\circ}$.

Thirdly, SWIM pitch and roll mispointing angles are also monitored and estimated through the level $1 \mathrm{~A}$ processing. The algorithm is based on the minimization of a cost function which computes the distance between the measured and a model echo power for a given measurement (at a given beam, antenna azimuth angle and elevation range). Both are corrected for geometric considerations, thermal noise and instrumental contributions, but not for the antenna gain. By minimizing the distance between measurement and model, we retrieve the mispointing angles in both the roll and pitch directions. Mispointing estimations obtained from this method (named method 1 hereafter) were compared to those obtained from two other methods: analysis of the difference between theoretical and measured roll and pitch angles of the platform (method 2), and analysis of cross-manoeuvers performed during two days in pitch and roll directions (method 3). Absolute mispointing angles estimated through these different methods are all of the order of $0.02^{\circ}$, i.e. one order of magnitude better than specified in the original requirements $\left(0.2^{\circ}\right)$. The angles derived from methods 1 and 2 are consistent: they show the same mean absolute mispointing angles of $0.02^{\circ}$ (averaged over of one cycle of data), and similar temporal variations. Figure 3 shows for method 1, the total mispointing angle (defined as $\left(\text { roll }^{2}+\text { pitch }^{2}\right)^{0.5}$ over several orbits. It highlights the impact of the reference ellipsoid, with oscillations of the total mispointing at the scale of the orbit (1.5 hour). Figure 4 illustrates the variation over a few minutes, of both roll (blue curve) and pitch (orange curve) mispointing angles. Oscillations visible on both angles show the impact of the antenna rotation. Results from method 2 give similar variations (not shown). As for the results from the cross-manoeuver method (method 3) they confirm the order of magnitude of the retrieved mispointing values but cannot be used to follow variations with the antenna rotation or along the orbit, as they provide only occasional estimates of roll and pitch mispointing angles. Thus, in practice, level 1A-estimation are used for monitoring and stability assessment of SWIM mispointing. It is also used as input of the nadir beam echo inversion (see section $\mathrm{V}$ ) and of the antenna gain pattern for the off-nadir beams (see section VI).

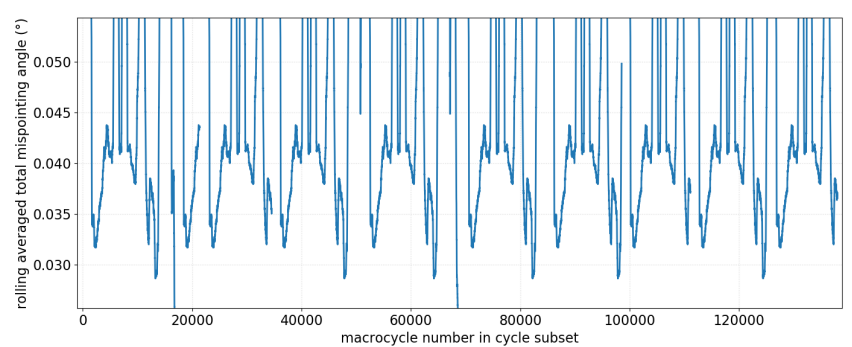

Figure 3: Rolling average of the total mispointing angles estimated in the level $1 \mathrm{~A}$ processing over several orbits of $1.5 \mathrm{~h}$ each, with respect to macrocycle number in the selected subset. Values are averaged over 500 macrocycles. The oscillations show the impact of the reference ellipsoid.

\section{NADIR BEAM PARAMETERS}

In a way similar to standard satellite altimeter missions, geophysical parameters from the nadir echo waveform over the ocean are inverted by applying a "retracking" algorithm based on the fit of a Brown model echo [16] to the recorded waveforms. For SWIM however, no information is provided on the epoch or height because CFOSAT is not an altimeter mission (no precise orbit determination, no microwave radiometry nor dual wavelength measurement for delay correction).

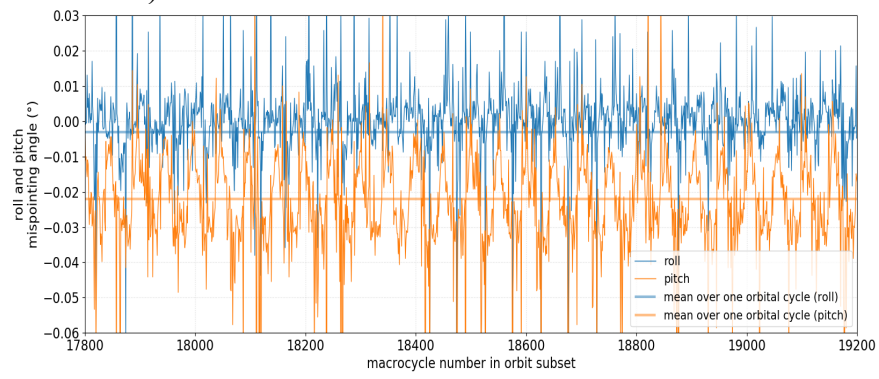

Figure 4: Zoom of roll (blue curve) and pitch (orange curve) mispointing angles estimated in the level $1 \mathrm{~A}$ processing over about 5 minutes. The thick blue and orange horizontal lines show the mean roll and pitch angles, respectively, averaged over one cycle of data (13 days).

Over ocean surfaces, the main geophysical products are thus the significant wave height, the normalized radar-cross-section $\sigma_{0}$, and the wind speed. An innovative algorithm, namely 'Adaptive retracking' [17] is implemented in the CFOSAT French ground segment. This "adaptive" algorithm has three specificities with respect to algorithms currently used for most of other altimeter missions:

- it considers the real point target response (estimated from on-board calibration sequences) instead of a theoretical function;

- the Brown model is determined taking mispointing values issued from level $1 \mathrm{~A}$ processing as inputs. This gives access to the mean-square slopes (mss) of the waves;

- the minimization is carried out according to a maximum likelihood criterion with the Nelder-Mead inversion method [18].

The retracking is performed at the rate of the nadir echo acquisition (every $220 \mathrm{~ms}$ in the nominal mode of SWIM acquisition), and the geophysical products are provided either 
as "native" values (at $4.5 \mathrm{~Hz}$ ), or as averaged values $(1 \mathrm{~s}, 4.5 \mathrm{~s}$ or per box). This Adaptive Retracking method shows remarkable results for all the retrieved parameters as detailed below.

\section{1) Significant Wave Height}

We have compared the significant wave height from the SWIM nadir observation, to collocated model data (ECMWF WAM and MFWAM). The result show a very weak and stable bias: about $3 \mathrm{~cm}$ with respect to ECMWF over a one orbital cycle period (see Fig. 5-6), and $1 \mathrm{~cm}$ with respect to MFWAM, over 3 months. As shown in Fig. 6, this bias is only slightly variable with wave height, and the dependency with wind is almost negligible. Figure 7 shows the comparison with Jason 3 data. It also highlights a very small bias, with a mean difference less than $1 \mathrm{~cm}$, and a standard deviation of $35 \mathrm{~cm}$. Same kind of results were obtained for comparison to AltiKa data, with a mean $\mathrm{Hs}$ difference of $6 \mathrm{~cm}$ w.r.t AltiKa and a standard deviation of $35 \mathrm{~cm}$.

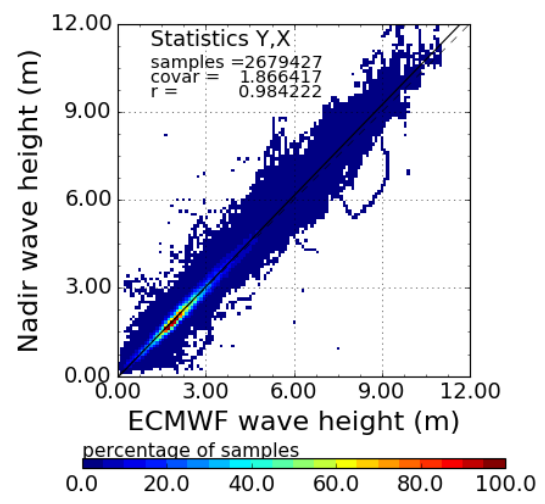

Figure 5: Significant wave height from SWIM nadir observations compared to ECMWF model data for a 13-day period (9-21 September 2019).

\section{2) Normalized radar cross-section}

The nadir normalized radar cross-sections from SWIM were compared to those provided at cross-over points by the altimetry missions Jason 3 and AltiKa. Figure 8 highlights a remarkable consistency with the Jason 3 data with a mean difference of about $0.25 \mathrm{~dB}$ w.r.t Jason 3 (std $0.6 \mathrm{~dB}$ ); the mean difference with the AltiKa Ka-Band instrument is around $2.5 \mathrm{~dB}$ (std $0.7 \mathrm{~dB}$ ) with latitude dependency, as expected due to the different operation frequency (Ku-Band vs Ka-Band).
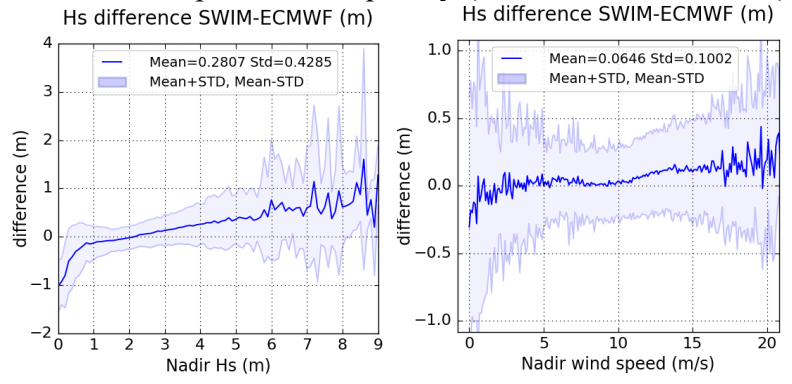

Figure 6: Difference between SWIM and ECMWF significant wave height. Left: as a function of significant wave height; right: as a function of wind speed. Blue/light blue: mean/one std envelope..

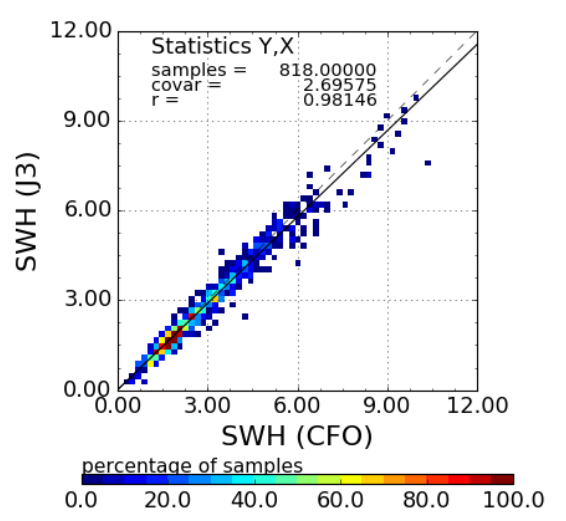

Figure 7: Significant wave height from SWIM nadir observations compared to Jason 3 altimeter values at crossover points for time difference less than 3 h (1 orbital cycle: 14-26 August 2019)

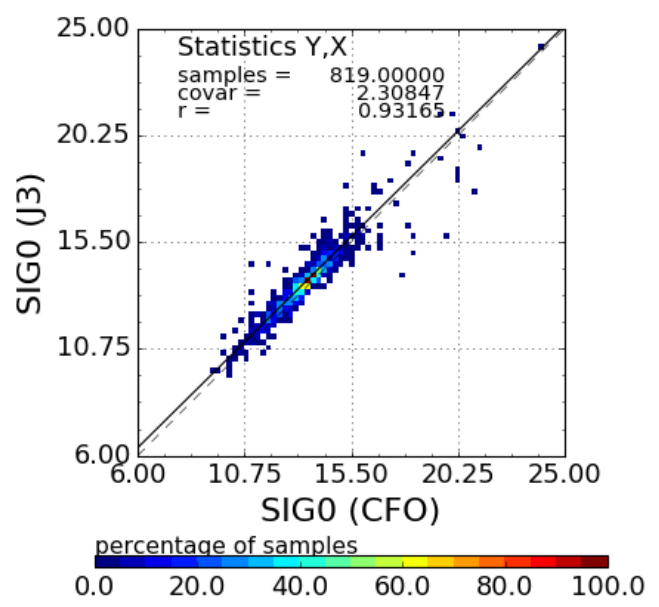

Figure 8: Same as Fig. 7 but for the nadir $\sigma_{0}$ values.

\section{3) Wind speed}

Nadir wind speeds provided in SWIM products are computed with the algorithm [19] used operationally for the Jason altimetry missions. These estimations are compared with the ECMWF model data in Fig. 9. The mission accuracy specification of less than $2 \mathrm{~m} / \mathrm{s}$ for wind speed is globally satisfied. However, the nadir sensor tends to overestimate low winds (below $8 \mathrm{~m} / \mathrm{s}$ ) and underestimate higher winds when one takes the model estimations as reference data. A study is underway to reduce such discrepancies by developing an adapted algorithm based on the nadir data themselves to better reflect their behavior. The algorithm will take the form of a $2 \mathrm{D}$ Look-Up-Table depending on the data pair $\left(\sigma_{0}, \mathrm{Hs}\right)$ as proposed in [20]. One year of data will be analyzed to ensure that a stable statistical model will be obtained and that all seasonal conditions have been observed between roughness, wind and wave parameters. 


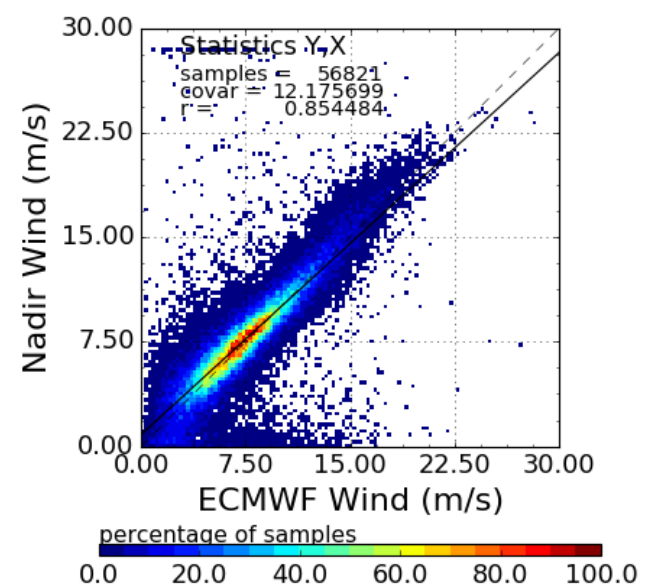

Figure 9: SWIM nadir wind speed compared to ECWMF wind speed for a 13-day period (9-21 September 2019)

In the nadir product, a rain flag is defined following the principle proposed in [21] and used for AltiKa data. This method was adapted to SWIM during the first months of the mission. The first results indicate a good consistency between the flags raised and rain provided by collocated radiometers (see [17]). The flag is also used to flag "bloom" events but some tuning is still necessary for this part.

One of the motivations for choosing the "Adaptive" retracking method was to reduce the impact of the relatively low nadir measurement rate of SWIM (at $4.5 \mathrm{~Hz}$ approximatively due to time spent on illuminating the off-nadir beams). An analysis was performed to qualify and quantify the improvement of adaptive retracking with respect to the conventional altimetry retracking algorithm based on the Maximum Likelihood Estimator MLE4. By using a spectral analysis of significant wave height series along-track, we found a $45 \%$ reduction of noise which allows to get the same performance in SWIM nadir product $(20 \mathrm{~cm}$ rms noise) for $4.5 \mathrm{~Hz}$ measurement rate and in Jason-3 official product $(20 \mathrm{~Hz}$ measurement rate). Significant improvements are also observed in the $\sigma_{0}$ restitution, as described in [17].

\section{NORMALIZED RADAR CROSS-SECTION OF OFF-NADIR BEAMS}

The normalized radar cross-section $\sigma_{0}$ is provided for all the SWIM beams from $0^{\circ}$ to $10^{\circ}$ and for all scenes (ocean, continent) as far as the nadir tracking has been successfully achieved with the on-board processing. At Level $1 \mathrm{~A}, \sigma_{0}$ is provided over the successive footprints (about $18 \mathrm{~km}$ long) with a discretization of $1.4 \mathrm{~m}$ (for the antenna beams $2^{\circ}$ and $4^{\circ}$ ), 0.7 $\mathrm{m}$ (for the antenna beam $6^{\circ}$ ), or $1 \mathrm{~m}$ (for the antenna beams $8^{\circ}$ and $10^{\circ}$ ). As SWIM is a Real Aperture Radar, $\sigma_{0}$ measurements refer to azimuthally-integrated quantities (over about $18 \mathrm{~km}$ perpendicularly to the line of sight). At Level $2, \sigma_{0}$ are provided as averaged values per bins of $0.5^{\circ}$ in incidence and $15^{\circ}$ in azimuth, and referenced in the geometry of the wave cells, with one box every $70 \mathrm{~km}$ (approximately) on each side of the nadir track (see next section for the geometry of the cells).

When converting the radar echo to $\sigma_{0}$ values, the classical radar equation is considered; it includes geometrical and radiometric corrections. For the geometrical corrections, the altitude of the satellite is provided by the epoch of the nadir beam of the same macrocycle. The mispointing angles estimated as explained in section IV are used to apply an antenna gain pattern compensation (azimuthally integrated value) which is taken from pre-calculated look-up tables parameterized as a function of incidence, azimuth angles, and roll and pitch angles. Finally, at level $1 \mathrm{~A}, \sigma_{0}$ values are corrected from the thermal noise by subtracting the mean value of the thermal noise estimated as explained in section IV. For the radiometric corrections (instrument gain and losses), the calibration coefficients from the most recent on-board calibration sequence is taken into account and the thermal noise mean level is subtracted. Note that no atmospheric correction is applied at Level 1A. In the opposite, Level 2 includes such a correction to account for attenuation by the dry and wet atmosphere (water vapor and liquid cloud water taken in auxiliary files generated from ECWMF model short term forecasts).

(a)

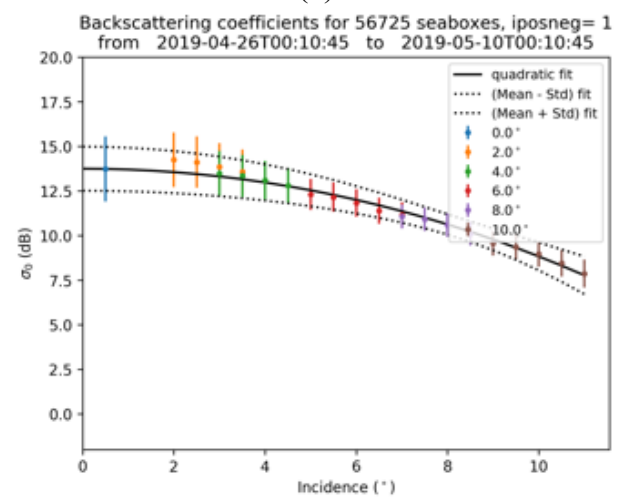

(b)

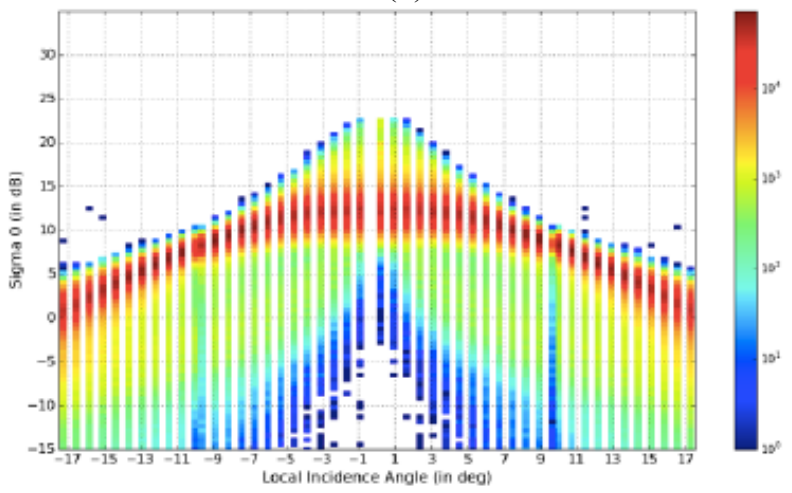

Figure 10: (a) SWIM mean profiles of $\sigma_{0}$ with incidence angle over the free ocean surface; (b) statistics of $\sigma_{0}$ from GPM/PR data for negative and positive incidence angles with respect to nadir $(N$. Longepe, personal communication).

Figure 10a illustrates the mean trend of $\sigma_{0}$ obtained from L2 products from the different beams (values averaged every $0.5^{\circ}$ in incidence and $15^{\circ}$ in azimuth). As shows Fig.10b, the mean trend is globally consistent with results provided by GPM data sets ([15]), see Fig.10b. The relative biases of $\sigma_{0}$ between the different SWIM beams is very small (of the order of 0.1 to 0.2 $\mathrm{dB}$ according to Fig. 10a) except between beams $2^{\circ}$ and $4^{\circ}$. 

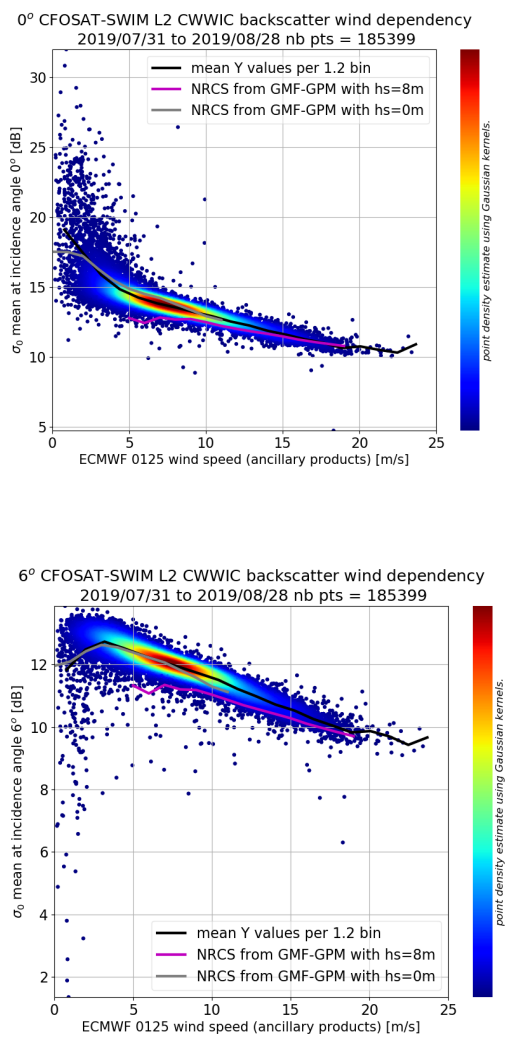
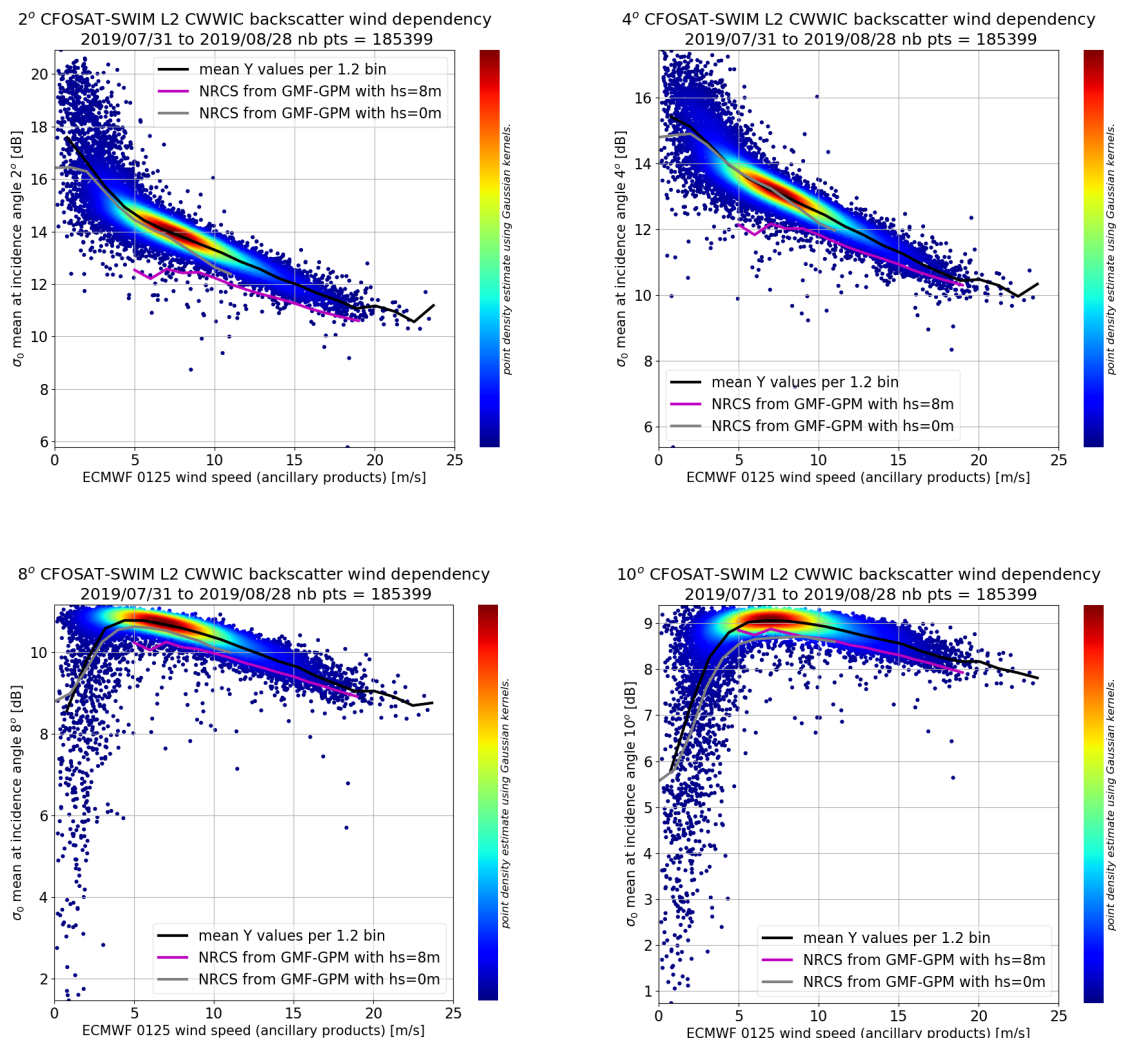

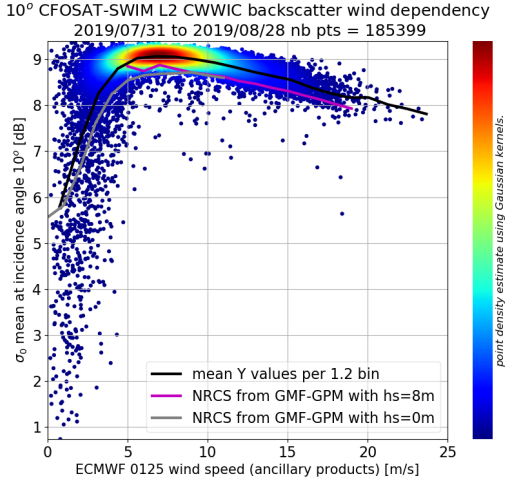

Figure 11: Distribution of $\sigma_{0}$ values at 0, 2, 4,6, 8 and $10^{\circ}\left( \pm 0.5^{\circ}\right)$ as a function of wind speed (taken from the ECMWF model). The black line shows the mean value. The color lines correspond to mean values of GPM data for the same wind and for significant wave heights of 0m (green) and $8 m$ (purple)

A detailed analysis is in progress to estimate more precisely the possible biases between the different SWIM beams. Figure 11 illustrates the dependence of $\sigma_{0}$ with wind speed. SWIM data are very consistent (less than $1 \mathrm{~dB}$ difference) with the GPM data mean trend. The sensitivity to wind speed is very small for the $10^{\circ}$ and $8^{\circ}$ beams $(1 \mathrm{~dB}$ to $1.5 \mathrm{~dB}$ difference between 5 and $20 \mathrm{~m} / \mathrm{s}$ ) and gradually increases at smaller incidence angles. Thanks to this smallest sensitivity of $\sigma_{0}$ with wind speed at 8 and $10^{\circ}$, the dominant effect in the $\sigma_{0}$ fluctuations at the scale of the footprint will hence be the tilt of the long waves, so that the best results for the wave inversion are expected to come from these incidence ranges. At light winds (typically less than 4 to $5 \mathrm{~m} / \mathrm{s}$ ), we can observe some outliers with low $\sigma_{0}$ values in Fig.11. Such outliers may come from non-standard scenes (for example affected by rain); note that it was chosen for the data analysis dedicated to the validation, as presented here, to keep all data without rejections. Starting mid-2020, the operational processor will be configured with additional quality controls to account for either the nadir rain flag or a flag specific for offnadir beams based on mean and standard deviations of $\sigma_{0}$.

\section{FLUCTUATION SPECTRA AND SPECKLE NOISE}

\section{A. Characterization of the fluctuation spectra}

During the verification phase, one of the first steps was to assess the properties of the fluctuation density spectra. These spectra are defined as the Fourier Transform of the auto-correlation function of the $\sigma_{0}$ fluctuations projected on ground within each footprint [2]. At first, the general behavior of these fluctuation spectra was evaluated by analyzing mean spectra. These latter are obtained for each SWIM beam, by averaging fluctuation spectra over several days and all geographical positions. So it accounts for all kinds of sea-state conditions. These averaged spectra are used here to qualify the wavelength and the azimuth ranges which are adequately imaged by SWIM. The left part of Fig. 12 displays such a mean spectrum, for the $10^{\circ}$ beam, based on an average over 6 days of observations on the ocean. On the right-hand side of Fig.12, it is shown the corresponding mean wave slope spectrum calculated from collocated WW3 model wave spectra (averaged over the same positions and dates). First, Fig. 12 shows that outside an angular sector of $\pm 15^{\circ}$ with respect to the satellite track, the characteristics of the mean fluctuation spectrum are similar to those of the mean WW3 slope spectrum, with the most energetic parts spanning over wavelengths from 125 to $450 \mathrm{~m}$ and direction around the $45^{\circ}$ in the mean. It also shows that within an angular sector of about $\pm 15^{\circ}$ on each side of the along-track direction, there is a strong increase in the energy level not related to the surface ocean waves. Very similar results were obtained for the $6^{\circ}$ and $10^{\circ}$ beams. The energy peak in the along-track direction is due to an increased noise in the directions where the Doppler bandwidth becomes relatively small (due to the geometry of observations). This causes a drastic drop of the number of independent samples [22] which increases the speckle noise. 

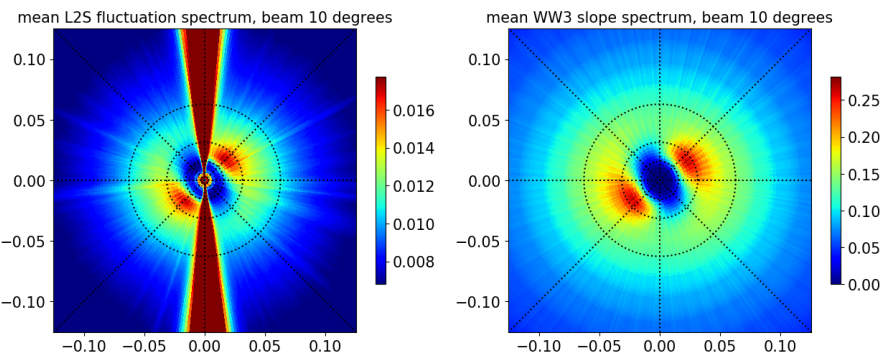

Figure 12: Mean fluctuation spectra for the period from April $25^{\text {th }}$ to April $30^{\text {th }} 2019$ (left) compared to the mean wave slope spectrum obtained from collocated points of WW3 outputs (right). Horizontal and vertical axes refer to the wave number of the waves in two orthogonal directions, with the vertical axis aligned with the satellite along-track direction.

This effect was anticipated before the satellite launch but its exact magnitude could not be simulated precisely. Figure 13 illustrates for all beams (from $2^{\circ}$ to $10^{\circ}$ ), in a polar representation, the correlation coefficient between the SWIM fluctuation spectra and the corresponding WW3 wave slope spectra, over the same time period as in Fig. 12. It shows that for beams $6^{\circ}, 8^{\circ}$ and $10^{\circ}$, the correlation coefficient is larger than 0.5 for all wavelengths greater than about $60 \mathrm{~m}$ and in all directions, except in the along-track sector affected by the increase of speckle noise. For beams $2^{\circ}$ and $4^{\circ}$, the correlation with WW3 is degraded compared to the other SWIM beams $\left(6^{\circ}\right.$, $\left.8^{\circ}, 10^{\circ}\right)$. This was expected, for the following reasons: i) no migration compensation is applied in the on-board processing for the signals coming from these beams, ii) the range resolution is less than for the other beams (see Table 2). Actually, these SWIM beams were not originally designed to retrieve ocean wave parameters, but they were designed mainly to provide mean radar cross-sections. Furthermore, at these near-nadir incidences, the relation between signal modulation spectra and wave slope spectra may become non-linear due to range bunching effects on the signal [23]. Nevertheless, Fig. 13 indicates that observations from these $2^{\circ}$ and $4^{\circ}$ SWIM beams contain information on waves while being more filtered than for the $6^{\circ}, 8^{\circ}$, and $10^{\circ}$ SWIM beams.

\section{B. Density spectrum of speckle noise}

As explained in $[2,6,7]$, the speckle noise must be taken into account in the inversion process. In the spectral domain, the spectral density of speckle must be subtracted from the fluctuation spectrum in order to obtain a spectrum related to the surface ocean waves (hereafter called modulation spectrum):

$$
P_{\delta \sigma 0}(k) \approx \delta(k)+P_{I R}(k) P_{m}(k)+P_{s p}(k)
$$

where $P_{\delta \sigma 0}$ is the density spectrum of the signal fluctuations. $P_{s p}$ is the density spectrum of the signal fluctuations due to speckle, $P_{m}(\mathrm{k})$ the density spectrum of signal modulations due to ocean waves, $P_{I R}$ is the density spectrum of the impulse response and $\delta$ is the Dirac function.

Assuming that the impulse response function follows a cardinal sine function in the temporal domain, both $P_{I R}(k)$ and $P_{s p}(k)$ follow a triangle shape, and $P_{s p}(k)$ is given by the following equation:

$$
P_{s p}(k)=\frac{1}{N_{\text {ind }}} \frac{\delta r}{2 \pi} \frac{1}{\sin \theta} \operatorname{tri}\left(\frac{k}{2 \pi} \frac{\delta r}{\sin \theta}\right)
$$

(a)

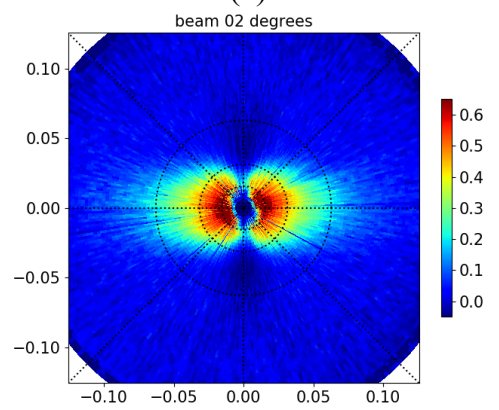

(c)

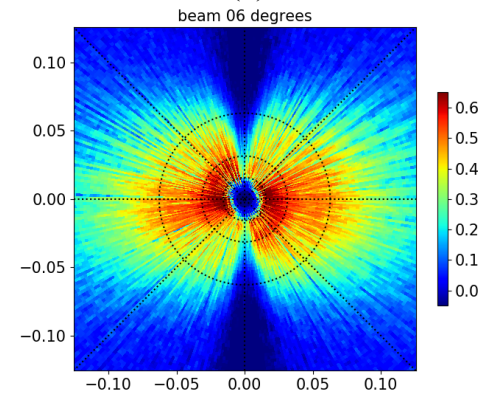

(b)

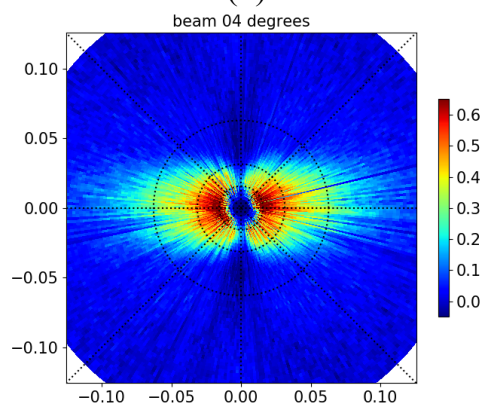

(d)

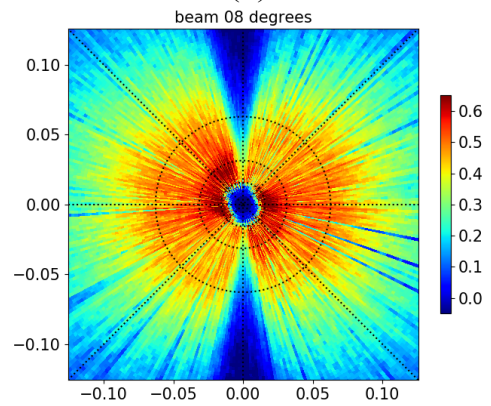

(e)

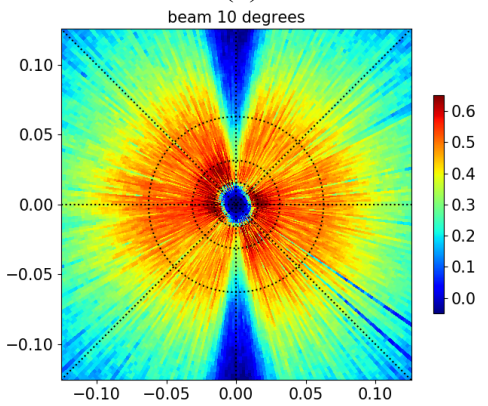

Figure 13: Polar plots of the correlation coefficient between SWIM fluctuation spectra and WW3 collocated wave slope spectra for the data set from April, $25^{\text {th }}$ to April, 30 $0^{\text {th }}$, 2019. Horizontal and vertical axes refer to the wave number of the waves in two orthogonal directions, with the vertical axis aligned with the satellite along-track direction. Panels ( $a, b, c, d, e):$ for SWIM beams $2^{\circ}, 4^{\circ}, 6^{\circ}, 8^{\circ}, 10^{\circ}$, respectively. 
where tri is the triangle function, $\mathrm{k}$ is the wavenumber, $\delta \mathrm{r}$ is the radar range resolution, $\theta$ the incidence angle, and $\mathrm{N}_{\text {ind }}$ is the number of independent samples.

Eq. (2) is different from Eq.13-15 in [2] because it now takes into account the real shape of the impulse response function. It follows a cardinal sine shape instead of a Gaussian shape as assumed in [2].

In the default option of the operational processor (used until mid-2020), the number of independent samples $\mathrm{N}_{\mathrm{ind}}$ was assumed to be the number of echoes integrated in real-time (Table 2), so that the speckle energy correction was isotropic. However, it is clear from the analysis shown in section VII-A (Fig. 12) that this latter assumption is not appropriate. Indeed, successive echoes are uncorrelated only if the Doppler bandwidth is much larger than the pulse repetition frequency (PRF), see [22]. When looking along-track, this assumption breaks down whereas other effects, which partially decorrelate successive echoes may play a role. In the initial version of the processing, the speckle was also estimated assuming the intrinsic radar resolution $(0.47 \mathrm{~m})$, without taking into account a possible reduction of speckle level brought by consecutive range bins averaging. Here, we present and use an empirical approach to estimate from the data themselves, the density spectra of the noise, on the one-hand outside, and on the otherhand inside the sector perturbed by the lack of radar Doppler bandwidth. Outside this sector, we hereafter call the analyzed spectra, the "background density spectrum of speckle".

\section{1) Background density spectrum of speckle}

Outside the sector impacted by the radar Doppler bandwidth reduction, we selected all fluctuation spectra from observations in directions which are the least affected by the ocean waves. They correspond to the direction of minimum fluctuation variances over each $180^{\circ}$ azimuthal sectors swept by the beam rotation. A mean speckle noise density spectrum was then calculated by averaging this result over a large number of spectrum samples in different sea-state conditions. Mean speckle noise density spectra were hence estimated with the same regular $\delta \mathrm{k}$ sampling as for the fluctuation spectra. In order to investigate the possible variation of this mean speckle spectral level with surface conditions or latitude, different classes of latitude and sea state were considered (classes identical to those used in section VII-B-2 below). The results indicate that the mean background speckle noise density spectrum does not depend significantly on sea-state conditions nor latitudes. This confirms that the background speckle is mainly dependent on the radar parameters and not on the surface conditions. Fig. 14 illustrates the results for one case, but the results are very similar for the other conditions. The figure shows that the dependence of the speckle density with wavenumber $\mathrm{k}$ is almost linear in the range of $\mathrm{k} 0.05$ to 0.3 $\mathrm{rad} / \mathrm{m}$, i.e. consistent with the linear model of Eq.2. It also indicates that compared to the theoretical spectra obtained from Eq.2 with theoretical values of $\mathrm{N}_{\text {ind }}$ (Table 2) and with $\delta \mathrm{r}$ $=0.47 \mathrm{~m}$ (dashed lines in color), the speckle level estimated empirically is higher, at least for $\mathrm{k}<0.25 \mathrm{rad} / \mathrm{m}$. When fitting Eq.(2) on the empirical spectra with $\mathrm{N}_{\text {ind }}$ and $\delta \mathrm{r}$ as free parameters (fit over wavenumbers in the [0.075-0.30 rad/m] range), we find larger $\mathrm{N}_{\text {ind }}$ and $\delta \mathrm{r}$ values compared to the theoretical values. This leads to values of the density spectrum at the origin $(\mathrm{k}=0)$ which are 2.5 (resp. 2.2, 1.9) larger than those corresponding to the theoretical values of $\delta \mathrm{r}$ and $\mathrm{N}_{\text {ind }}$ for beam $6^{\circ}\left(8^{\circ}\right.$, and $10^{\circ}$ respectively).

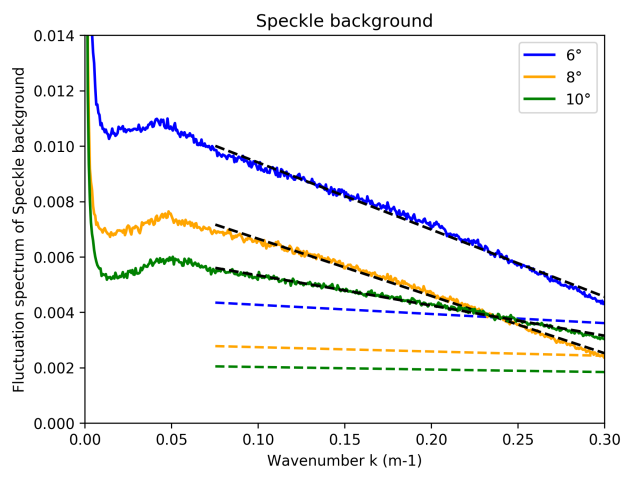

Figure 14: Mean background density spectra as a function of wave number $(\mathrm{rad} / \mathrm{m})$ for beams $6^{\circ}$ (solid blue), $8^{\circ}$ (solid orange) and $10^{\circ}$ (solid green). Dashed black lines correspond to the triangle-shaped model fitted on these spectra (adjustment between $k=0.075$ and 0.3

rad $\left.\mathrm{m}^{-1}\right)$. Dashed colored lines correspond to Eq.2 with the theoretical values of $N_{\text {ind }}$ and $\delta r$. This figure corresponds to data selected over a 7-day period (26 April-1 May 2019) in the [-10, $10^{\circ} \mathrm{J}$ latitude range and for surface conditions with Hs less than $2 \mathrm{~m}$ and wind speed less than $5 \mathrm{~m} / \mathrm{s}$ ).

This ratio is close to the number of range gates averaged during the on-board processing (see Table 2), which was not taken into account in the theoretical speckle estimates although it contributes to diminish the speckle spectral level. Note also that the larger $\delta$ r values estimated from our empirical approach yield a faster decrease with wave number of the speckle level compared to the theoretical case (see Fig.14). Combined with the different value at the origin, the two estimates of speckle level (theoretical and empirical) converge at large wavenumbers, around $0.2-0.3 \mathrm{rad} / \mathrm{m}$.

\section{2) Density spectrum of noise close to the along-track direction}

In order to build an empirical model for the noise density spectrum close to the along-track direction, the approach was to analyze the fluctuation spectra as a function of wavenumber over a sector of $\pm 30^{\circ}$ from the along-track direction, and its dependence on geometry of observation (latitude, look direction, orbit orientation) and sea-state. 21 classes of data were selected for each SWIM incidence conditions $\left(6^{\circ}, 8^{\circ}, 10^{\circ}\right)$, composed of 7 classes of latitude $\left(\left[-70^{\circ},-50^{\circ}\right],\left[-50^{\circ},-30^{\circ}\right]\right.$, [$\left.\left.30^{\circ},-10^{\circ}\right],\left[-10^{\circ}, 10^{\circ}\right],\left[10^{\circ}, 30^{\circ}\right],\left[30^{\circ}, 50^{\circ}\right],\left[50^{\circ}, 70^{\circ}\right]\right)$, and 3 classes of combination of wind speed $U$ and significant wave height $(U<5 \mathrm{~m} / \mathrm{s}$ and $\mathrm{Hs}<2 \mathrm{~m}, 5<U<9 \mathrm{~m} / \mathrm{s}$ and $\mathrm{Hs}<2 \mathrm{~m}$, and $U>9 \mathrm{~m} / \mathrm{s}$ and $2<\mathrm{Hs}<4 \mathrm{~m}$ ). We choose the sea-surface classes so as to minimize the contributions of waves in the analyzed fluctuation spectra, while keeping enough samples to ensure a high statistical significance.

Figure 15 shows the resulting mean spectra as a function of wave number (with the same wavenumber sampling as the 
fluctuation spectra), for the $10^{\circ}$ SWIM beam and for one of these classes $\left(\left[-10^{\circ}\right.\right.$ to $\left.10^{\circ}\right]$ in latitude and $\left.\mathrm{U}<5 \mathrm{~m} / \mathrm{s}\right)$. Solid lines with different colors are plotted for different directions of observation with respect to the satellite track. The results indicate that for azimuth angles from $0^{\circ}$ to about $15^{\circ}$ with respect to the satellite track, the shape and level of the spectrum change considerably, with energy decreasing significantly from $0^{\circ}$ to $15^{\circ}$ at all wave numbers. It stabilizes for azimuth angles larger than $15^{\circ}$ (see the purple curve in Fig. 15). At these azimuth values, its level and shape become very similar to the background mean speckle spectrum, determined away from the along-track direction, as described in section VII-B-1, and identified by the black solid line in Fig. 15. Fig. 15 also illustrates that the increase of energy with respect to the background level is larger at small wave numbers, in the sector affected by the loss of Doppler bandwidth. These trends are similar for the other SWIM beams. However, the increase of the along-track fluctuation spectra with respect to the background speckle is much higher for the $6^{\circ}$ beam than for the $8^{\circ}$ and $10^{\circ}$ beams. The trends with latitude and surface conditions are discussed later on in this section.

Our analysis led us to model the density spectrum of noise in this sector affected by the Doppler bandwidth reduction, as the sum of a $2^{\text {nd }}$ order polynomial $\left(\mathrm{P}_{\mathrm{sp}}(\mathrm{k})=\mathrm{a} \mathrm{k}^{2}+\mathrm{b} \mathrm{k}+\mathrm{c}\right.$ ) (to represent the trend with $\mathrm{k}$ as shown in Fig.15) and of a triangle function to account for the background noise discussed in the above section VII-B-1. Each coefficient of the $2^{\text {nd }}$ order polynomial was then assumed to vary linearly with the azimuth angle:

$$
\mathrm{a}=\mathrm{a} 0\left(\phi-\phi_{0}\right)+\mathrm{a}_{1}, \mathrm{~b}=\mathrm{b}_{0}\left(\phi-\phi_{0}\right)+\mathrm{b}_{1}, \mathrm{c}=\mathrm{c}_{0}\left(\phi-\phi_{0}\right)+\mathrm{c}_{1}
$$

where $\phi_{0}$ is the azimuth angle at which $\mathrm{P}_{\mathrm{sp}}(\mathrm{k})$ was observed to be maximum.

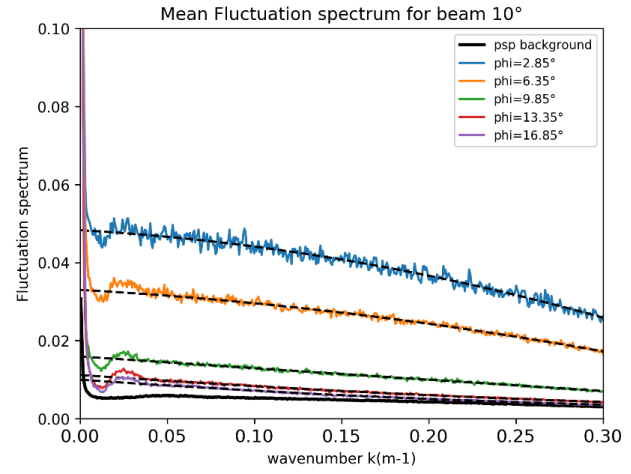

Figure 15: Color lines: Mean fluctuation spectra for the SWIM beam $10^{\circ}$, obtained by accumulating data over different azimuthal angles

(from about 2 to about $17^{\circ}$, w.r.t. the along-track direction, see insert legend) and over 6 days of observations ( $26^{\text {th }}$ April - $1^{\text {st }}$ May 2019). The dashed black lines represent the polynomial fit (estimated for $\mathrm{k}$ within [ 0.075 to 0.3$] \mathrm{rad} / \mathrm{m}$. The solid black line is the mean background speckle spectrum. See text for details.

We estimated $\phi_{0}$ for different geometrical conditions, by analyzing the fluctuation spectra level $\mathrm{P}_{s p}(\mathrm{k})$ at $\mathrm{k}=2 \pi / 30 \mathrm{rad} / \mathrm{m}$, in order to minimize the impact of long waves. It was found that $\phi_{0}$ depends on i) latitude, with $\phi_{0}$ almost aligned with the along-track direction at high latitudes, and shifted to about $3.5^{\circ}$ to $4^{\circ}$ from the along-track direction at latitude $0^{\circ}$, ii) track orientation (changing sign between ascending or descending) and iii) look direction (uptrack or downtrack). All these dependencies have been taken into account in our model. Note that they are consistent with the fact that the effective Doppler bandwidth is impacted by these geometrical parameters, due to Earth rotation.

The dependence of the noise spectral level with both latitude and sea-state conditions was analyzed, based on the 21 pre-cited classes of data composed of 7 classes of latitude, and 3 classes of surface conditions. Figure 16 illustrates, for each SWIM beam, and for the three different classes of surface conditions, the trend of the energy level estimated with our empirical model at $\mathrm{k}=0.1 \mathrm{rad} / \mathrm{m}$ and $\phi=\phi_{0}$, with respect to latitude. Results for other wavenumbers show the same trends. The figure first illustrates that the spectral level in the direction of maximum of speckle energy decreases from the $6^{\circ}$ beam to the $8^{\circ}$ and $10^{\circ}$ beams, as in the case of the background speckle noise (Fig 14). It also shows that for the three beams $\left(6^{\circ}, 8^{\circ}, 10^{\circ}\right)$, the impact of sea conditions is significant for high wind speed conditions $(\mathrm{U}>9 \mathrm{~m} / \mathrm{s})$, with a decrease of spectral level of $10 \%$ with respect to low and medium wind conditions $(\mathrm{U}<5 \mathrm{~m} / \mathrm{s})$. Hence it appears that in these conditions where decorrelation is not ensured by the radar acquisition conditions, wind speed starts to play a role on the decorrelation of echoes (hence on the noise spectrum) for wind intensities larger than about $9 \mathrm{~m} / \mathrm{s}$. The decrease of the spectral level of noise with increasing values of wind speed is compatible with a correlation time of the surface scatters decreasing with wind speed [24]. Finally, Fig.16 indicates also that the variation with latitude is significant for beams $6^{\circ}$ and $8^{\circ}$.

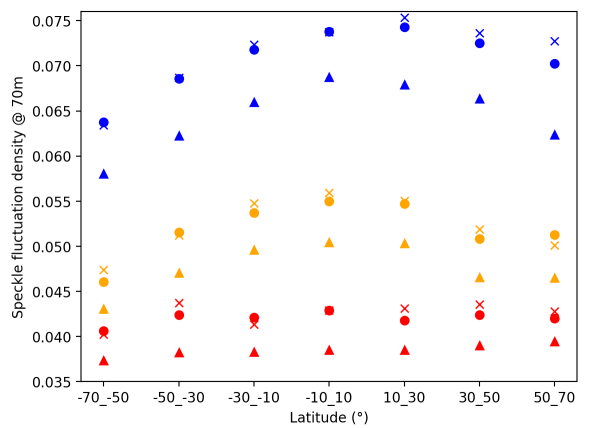

Figure 16: Speckle noise spectral level at $k=0.09 \mathrm{rad} / \mathrm{m}$ (wavelength of $\sim 70 \mathrm{~m}$ ), calculated from our empirical formulation, at the azimuth angle of maximum speckle level $\left(\phi=\phi_{0}\right)$. The results are plotted for the three SWIM beams in blue $\left(6^{\circ}\right)$, orange $\left(8^{\circ}\right)$, red $\left(10^{\circ}\right)$ and for three sea surface conditions (circle: $H_{s}<2 m \& U<5 \mathrm{~m} / \mathrm{s}$, cross: $H s<2 m \& 5<U<9 m /$, triangle: $2 m<H s<4 m \& 5<U<9 m / s)$.

\section{3) Impact of speckle noise correction}

Based on the analysis presented in sections VII-B-1 and VIIB-2), we applied the empirical speckle correction using 1) a triangular-shaped model outside the along-track direction and 2) a combination of the triangular-shaped model and a quadratic-shaped model in the azimuthal sectors up to $15^{\circ}$ around the along-track direction. Fig. 17a and 17d show two examples of directional wave spectra obtained by using this noise correction. They are compared to the co-located 
MFWAM spectra (Fig. 17b and 17e), and to the wave spectra obtained when using the original speckle model as implemented in the operational processing until mid-2020 (Fig 17c-17f). In the first example (Fig.17a-b-c), illustrating complex mixed sea conditions in the North Pacific (see Fig 17b), the new correction allows to detect the dominant wave systems, including the one propagating the along-track direction. With the initial processing this latter wave system was completely hidden by the noise signature. Also, the background noise is decreased with respect to the case with the initial speckle correction. In the second example (Fig 17a-b-c), where the dominant waves propagate across-track, the impact of the new correction with respect to the initial one is less important. (a)

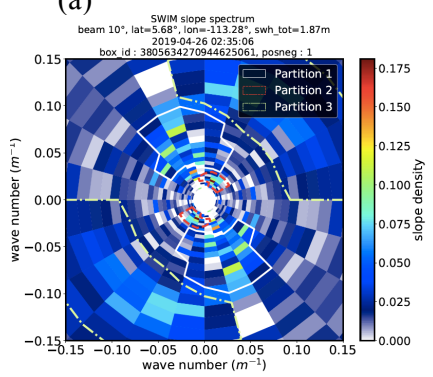

(b)

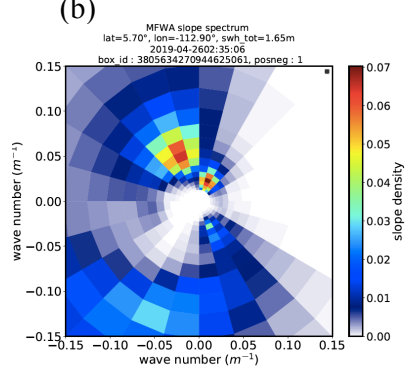

(c)

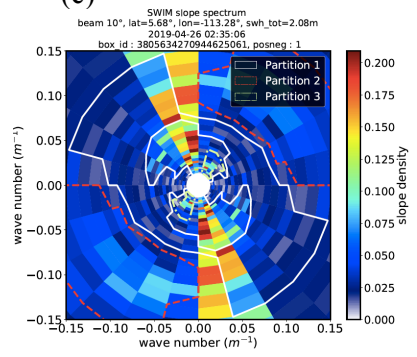

(d)

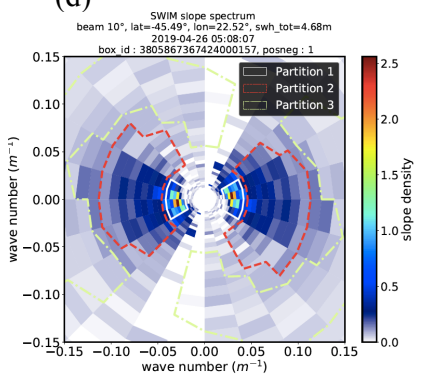

(e)

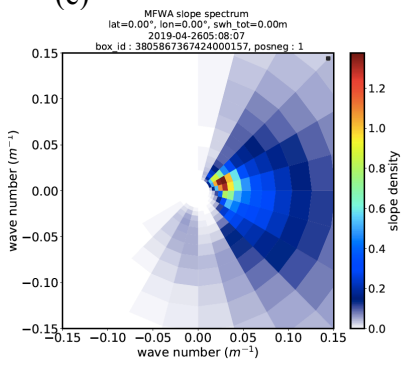

(f)

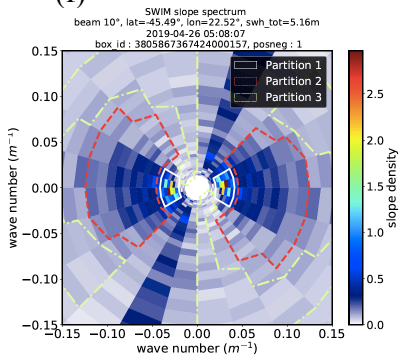

Figure 17: Examples of wave spectra from the $10^{\circ}$ beam observations with the speckle correction from the models presented in this paper $(a, d)$ and the original correction $(c, f)$. $(b, e)$ show the corresponding MFWAM wave slope spectra. The horizontal and vertical axes refer to Est-West and North-South directions,

respectively. The white, red and yellow contours on the SWIM spectra indicate the partitions (see section VIII). Date and position of observations are April 26 $201902: 35$ UTC at [5.68N,113.27W] for (a-c) and April 26 2019 05:08UTC at [45.49S,22.51E] for (d-f)

Nevertheless, it consistently decreases the background noise and suppresses the maximum of energy along track. It has to be recalled that in order to mitigate the problem of insufficient elimination of the speckle noise in the initial version of the SWIM data processing, a mask was applied in the along-track sectors on the wave spectrum. With this improved noise correction, the mask is not used anymore.

\section{WAVE SPECTRA AND ASSOCIATED PARAMETERS}

The Level 2 products are mainly the wave spectra and their parameters. They are estimated from the L1b data according to the following main steps:

- the modulation spectra are estimated in each look direction by subtracting a speckle noise spectrum to the fluctuation spectra. In this paper, the speckle correction is carried out according to the empirical model presented in section VII; i.e with the version implemented in the operational processing chain (version 5.0), from mid-2020;

- the modulation spectra are resampled in wave number; for the results presented here, 32 wave numbers in the range [0.0126-0.279] $\mathrm{rad} / \mathrm{m}$, corresponding to the wavelength domain $\sim[22-500] \mathrm{m}$;

- the modulation spectra are transformed into wave slope spectra by applying a « Modulation Transfer Function » (MTF) which uses an estimate of the mean falloff of $\sigma^{0}$ with incidence angle, in each azimuth direction (see [2]);

- the different directions of the wave spectra are then combined to build the polar 2D directional wave spectra at the scale of "wave box" of about $70 \mathrm{~km} \mathrm{x} 90 \mathrm{~km}$ (in the nominal mode of SWIM acquisition);

- a partition scheme based on the watershed algorithm as in [25] is applied to detect up to 3 partitions;

- wave spectral parameters (significant wave height, dominant direction and dominant wavenumber) are calculated on the polar spectrum and on its partitions.

\section{A. Global analysis of wave spectra and wave parameters}

A global analysis of the wave spectra quality was carried out by comparing at the global scale, wave spectra from SWIM and from the MFWAM wave model. The MFWAM model was chosen because in the context of the CFOSAT CAL/VAL, the forecasted wave spectra are collocated in near-real time by Météo-France on a systematic basis. Two kinds of analysis are presented hereafter:

- analysis of the full spectral content and parameters: correlation index between spectra, main parameters of the full wave spectra

- analysis of the main parameters associated with the partitions of the wave spectra

The main parameters which are analyzed hereafter are the significant wave height Hs, the dominant wavelength and the dominant direction. From SWIM they are calculated by the operational processor on the 2D polar wave slope spectra. For MFWAM, the wave parameters provided as output of the Météo-France operational processor are converted to take into account the conversion of peak period to peak wavelengths, assuming the deep water dispersion relationship. Note that for the spectral partitions, the MFWAM model provides mean periods and mean directions, whereas the parameters estimated on the SWIM data are dominant wavenumbers and direction. This might explain some slight differences in the comparison below (section VIIIA-2). 


\section{1) Analysis of the full spectra content and parameters}

Following [26], a first step was to calculate a 2D correlation index either between pairs of spectra themselves, or between spectra from each SWIM beam and the MFWAM spectra. The results are presented in Table 7 . The correlation index between spectra from pairs of SWIM beams is better than 0.5 for at least $70 \%$ of the cases with the highest correlations between beams $8^{\circ}$ and $10^{\circ}$. The results also indicate that the $6^{\circ}$ beam behaves slightly differently from the other beams. When correlation indexes are compared to Hs values, it appears that for all SWIM pairs, the highest values of the correlation index are observed for Hs larger than about $2 \mathrm{~m}$. As for the correlation index between spectra from SWIM beam and MFWAM, Table 7 indicates that the correlation index is better than 0.5 for $68 \%$ (respectively $57 \%$ and $39 \%$ ) of cases, for the $10^{\circ}$ beam (respectively for beams 8 and $6^{\circ}$ ). These numbers indicate that the spectra from beam $6^{\circ}$ are significantly less comparable to MFWAM spectra than those from beams $8^{\circ}$ and $10^{\circ}$. The low correlation values (less than 0.5 ) between the $6^{\circ}$ beam spectra and MFWAM spectra mainly occur in regions with small Hs values (typically less than the order of $1 \mathrm{~m}$ ).

\begin{tabular}{|l|c|}
\hline \multicolumn{1}{|c|}{ Pairs of spectra } & $\begin{array}{c}\% \text { of cases } \\
\text { with R }>0.5\end{array}$ \\
\hline SWIM 6/ SWIM 10 & 71 \\
\hline SWIM 6/ SWIM 8 & 70 \\
\hline SWIM 8 / SWIM 10 & 85 \\
\hline SWIM 6 / MFWAM & 39 \\
\hline SWIM 8 / MFWAM & 57 \\
\hline SWIM 10 / MFWAM & 68 \\
\hline
\end{tabular}

Table 7: Percentage of pairs of spectra which exhibit a correlation index higher than 0.5. The correlation index is calculated according to [26]. The data set is from April $26^{\text {th }} 2019$ to May $9^{\text {th }} 2019$.

Figure 18 illustrates the scatter plots of the SWIM-beam $10^{\circ}$ parameters versus MFWAM parameters estimated on the full spectra. The first row of Table 8 gives the corresponding statistical scores. For the significant wave height, the correlation is very high (0.97) and the rms difference quite small $(0.26 \mathrm{~m})$, but the mean trend is characterized by a slope of 0.82 . This yields an overestimation of Hs at small values $(\mathrm{Hs}<3 \mathrm{~m})$ and underestimation at large values $(\mathrm{Hs}>3 \mathrm{~m})-$ see Fig.18a. For the 13-days period analyzed here, most of the overestimated Hs values are located in the Southern part of the oceans, in the region of high wind speed and high significant wave height. Very similar results were obtained in terms of bias for beams $6^{\circ}$ and $8^{\circ}$, but with a slightly larger rms difference ( 2 to $4 \%$ increase). For the direction, the agreement is good except in the direction interval $\left[170^{\circ}\right.$ $195^{\circ}$ ] from North, and its $180^{\circ}$ modulo counterpart (see also Fig. 18b). We verified that this is due to limitations in the detection of waves propagating in the along-track direction, which occur in spite of our noise correction improvement. For the dominant wavelength, a high correlation coefficient is observed, however slightly less than in the case of Hs. Fig. $18 \mathrm{c}$ indicates that peak wavelengths as short as $70 \mathrm{~m}$ are detected on the SWIM spectra, a threshold which is compliant with our initial specifications. The few outliers which can be seen in Fig. 18c correspond to SWIM wavelengths underestimated with respect to MFWAM, when MFWAM wavelengths are larger than about $250 \mathrm{~m}$.
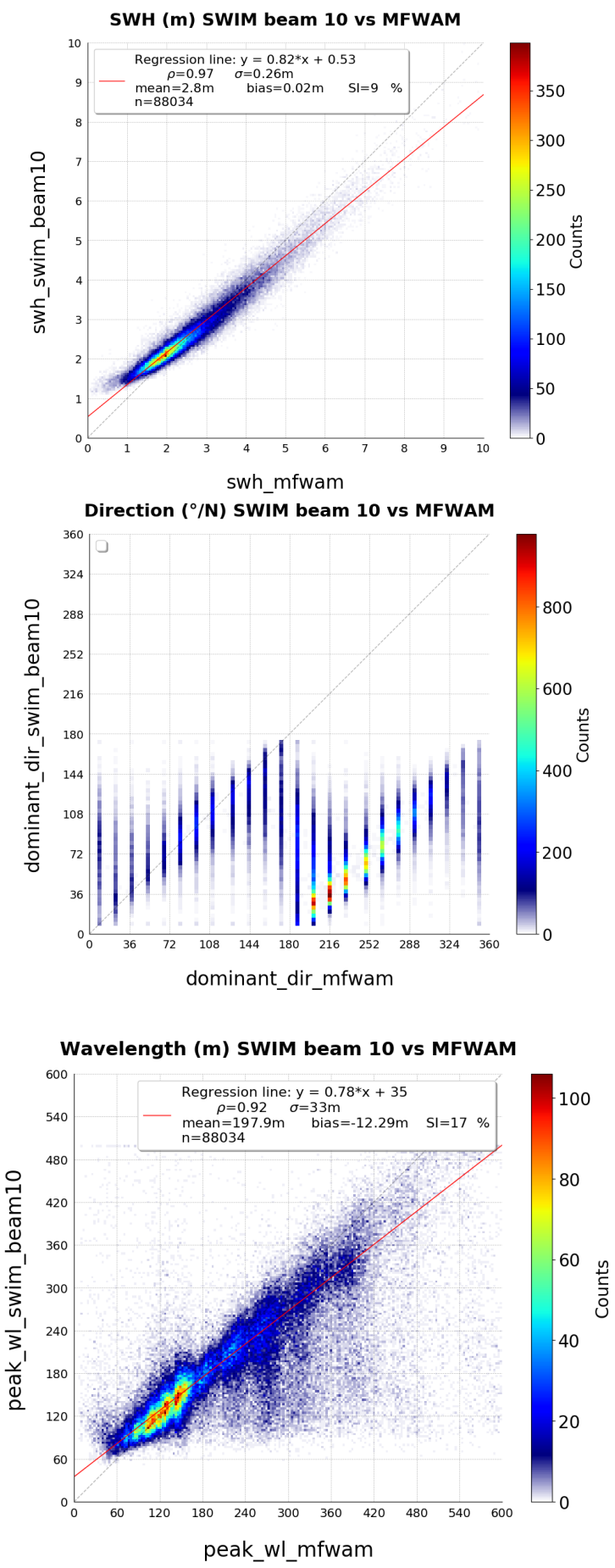

Figure 18: Scatter plots of SWIM parameters from the $10^{\circ}$ beam full spectra as a function of MFWAM full spectra parameters, for a 13 days period (26 April-8 May 2019)- (a) Significant wave height Hs; (b) Dominant direction (from North; (c) Dominant wavelength. The color code represents the number of points per bin of values. The red line on (a) and (c) represents the linear fit. 
Most of these points are associated with along-track wave propagating waves, which are also identified as outliers in Fig. 18b. Overall, the same conclusions were reached on these 3 parameters with data from SWIM beams $8^{\circ}$ and $6^{\circ}$. However, an increased effect of the above mentioned limitations is noted for observations from the $6^{\circ}$ beam. This is likely due to a higher impact of speckle at this incidence with respect to the other beams.

\section{2) Analysis of wave parameters from partitions}

Analyzing parameters from wave partitions is important because these parameters are those used in the assimilation of wave spectral observations for wave models [27]. This is also the information required to study the spatio-temporal evolution of wave fields with an approach as the one proposed by [28]. For the objective of SWIM data validation, considering partitions instead of the full spectrum is a way to better focus the analysis on the parameters of different wave systems. It also minimizes the possible contribution of the background noise, because the contours of partitions exclude a large part of the noise floor. On the other hand, comparing parameters of wave partitions requires to adequately associate wave partitions from the two sets. In a first step, we associated the most energetic partition of SWIM spectra (called $1^{\text {st }}$ SWIM partition) to the most energetic swell detected by MFWAM (called $1^{\text {st }}$ swell from MFWAM). In both cases, partitioning was carried out by using the classical watershed algorithm [25], but for SWIM spectra we did not use any additional assumption to classify the partitions as swell or wind-sea. In opposite, partitions identified in output of the MFWAM model, are classified into two swell systems and one wind-wave system. For this latter category, a criterion on wave age is used based on the ratio of $U / C_{p}$ where $\mathrm{U}$ is the wind speed of the model and $\mathrm{C}_{\mathrm{p}}$ the phase velocity of the partition wavelength projected in the wind direction. We verified that in the analyzed data set, the $1^{\text {st }}$ swell partition of MFWAM is the most energetic one among the three MFWAM partitions for $80 \%$ of the cases, and thus in $80 \%$ of the cases, the simple SWIM/MFWAM association of partitions as described just above is fully justified. Comparisons on wave parameters are illustrated in Fig. 19 and the scores are given in the second line of Table 8. In addition, geographical maps of the same parameters are illustrated in Fig.20. Although the statistical parameters indicate generally lower scores with respect to the comparison carried out for the full spectra parameters, we still observe a rather good consistency between SWIM and MFWAM parameters. For the wave heights of the $1^{\text {st }}$ SWIM partition compared to the $1^{\text {st }}$ swell of MFWAM, we find the same trend as in Fig.18, with an overestimation of SWIM with respect to MFWAM for the smallest wave heights $(<1.5 \mathrm{~m})$ and an underestimation for the largest ones. Underestimation of the SWIM partition energy with respect to MFWAM occurs in the southern hemisphere, in the region of high Hs (Fig. 20 a, d). The results are similar for beams $6^{\circ}$ and $8^{\circ}$ with however a slightly larger dispersion (rms 0.48 and 0.49 $\mathrm{m}$, respectively compared to $0.40 \mathrm{~m}$ for beam $10^{\circ}$ ). As for the direction, the global scores are clearly impacted by inconsistent wave directions found in the sector [170-195] degrees from North (modulo $180^{\circ}$ ) which are the directions corresponding roughly to the along-track directions (see also Fig.20c,f). But outside this interval the correspondence between SWIM and MFWAM directions is excellent with almost no bias. For the wavelengths (Fig 19c), the bias remains small (less than $30 \mathrm{~m}$ ) at all wavelengths but we also observe two groups of outliers. The first one, for SWIM wavelengths between 60 and $120 \mathrm{~m}$ correspond to the along-track propagating waves (see also Fig. 20). The outliers are probably related to an incorrect
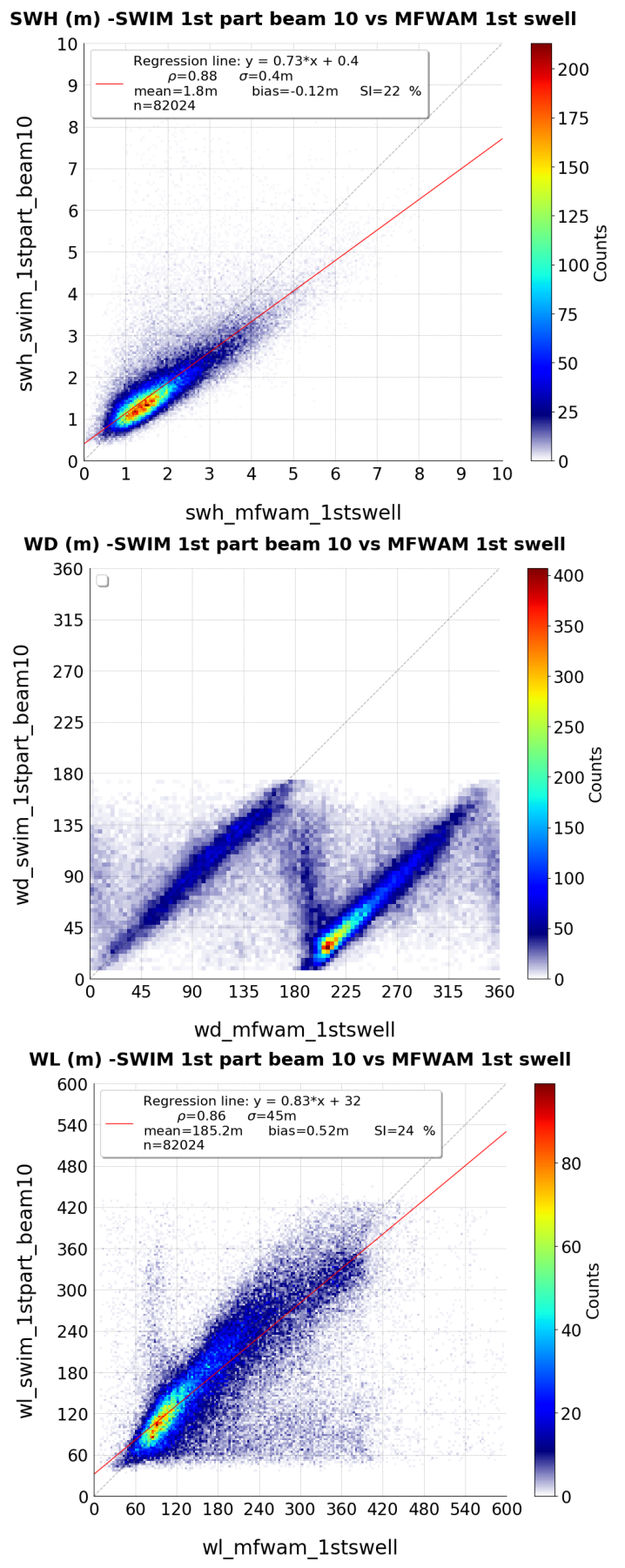

Figure 19: Same as Fig.18, but for the parameters of the $1^{\text {st }}$ partition if the SWIM spectra (beam $10^{\circ}$ ) compared to the parameters of the $1^{\text {st }}$ swell of MFWAM 


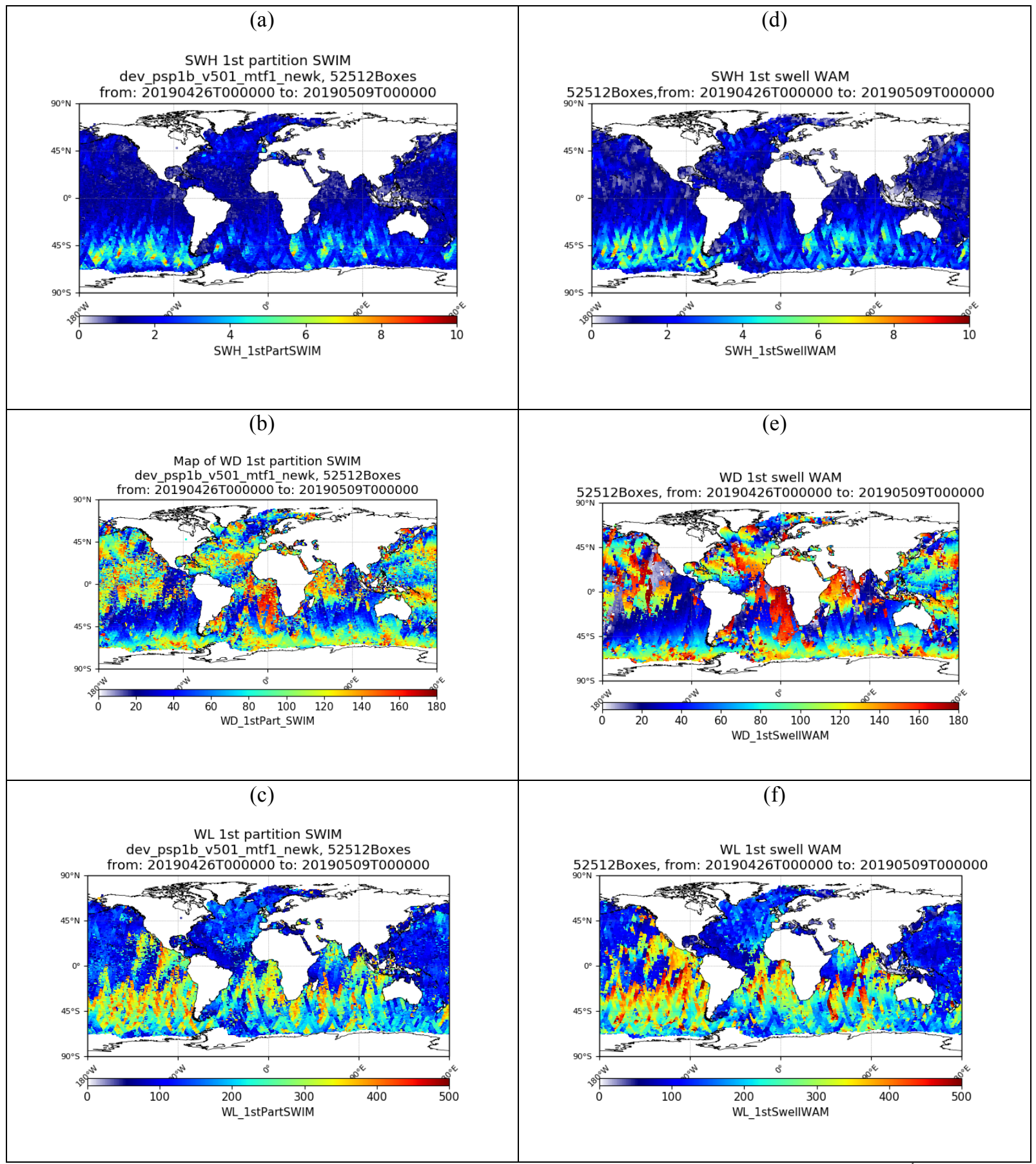

Figure $20:$ Maps of SWIM $(a, b, c)$ and MFWAM $(d, e, f)$ parameters for a period of about 13 days starting on April $26^{\text {th }} 201902: 00$. a and d: wave height of the first partition; $b$ and $e$ : direction of the first partition (for MFWAM the directions have been folded in the [0-180 ${ }^{\circ}$ interval to be compared with the SWIM data); c and f: wavelength of the first partition. The SWIM results are shown for the $10^{\circ}$ incidence beam. See text for details. 


\begin{tabular}{|c|c|c|c|}
\hline & $\begin{array}{c}\text { Waveheight } \\
(\mathrm{m})\end{array}$ & $\begin{array}{c}\text { Wavelength } \\
(\mathrm{m})\end{array}$ & $\begin{array}{c}\text { Direction } \\
\left({ }^{\circ}\right)\end{array}$ \\
\hline $\begin{array}{l}\text { SWIM versus } \\
\text { MFWAM- full } \\
\text { spectra }\end{array}$ & $\begin{array}{l}0.82 \mathrm{x}+0.53 \\
\text { CC: } 0.97 \\
\text { MB: } 0.02 \mathrm{~m} \\
\mathrm{rms}: 0.26 \mathrm{~m} \\
\text { SI: } 9 \%\end{array}$ & $\begin{array}{l}0.78 \mathrm{x}+35 \\
\text { CC: } 0.92 \\
\text { MB: }-12.3 \\
\text { rms : } 33 \mathrm{~m} \\
\text { SI } 17 \%\end{array}$ & $\begin{array}{l}0.59 x+43 \\
\text { CC : } 0.69 \\
\text { MB: } 3.9^{\circ} \\
\text { rms: } 26^{\circ}\end{array}$ \\
\hline $\begin{array}{ll}\text { SWIM } & 1^{\text {st }} \\
\text { partition } & \text { versus } \\
\text { MFWAM } & 1^{\text {st }} \\
\text { swell }\end{array}$ & $\begin{array}{l}0.73 \mathrm{x}+0.40 \\
\text { CC: } 0.88 \\
\mathrm{MB}:-0.12 \mathrm{~m} \\
\mathrm{rms}: 0.40 \mathrm{~m} \\
\text { SI: } 22 \%\end{array}$ & $\begin{array}{l}0.83 \mathrm{x}+32 \\
\text { CC: } 0.86 \\
\text { MB: } 0.5 \mathrm{~m} \\
\text { rms : } 45 \mathrm{~m} \\
\text { SI: } 24 \%\end{array}$ & $\begin{array}{l}0.57 \mathrm{x}+35 \\
\mathrm{CC}: 0.72 \\
\mathrm{MB}: 1.0^{\circ} \\
\mathrm{rms}: 27^{\circ}\end{array}$ \\
\hline $\begin{array}{l}\text { SWIM partition } \\
\text { matched to } \\
\text { MFWAM } 1^{\text {st }} \\
\text { swell partition }\end{array}$ & $\begin{array}{l}0.78 \mathrm{x}+0.18 \\
\text { CC: } 0.89 \\
\text { MB: }-0.22 \mathrm{~m} \\
\mathrm{rms}: 0.38 \mathrm{~m} \\
\text { SI: } 23 \%\end{array}$ & $\begin{array}{l}0.85 \mathrm{x}+35 \\
\text { CC: } 0.90 \\
\text { MB: } 5.6 \mathrm{~m} \\
\text { rms : } 38 \mathrm{~m} \\
\text { SI: } 19 \%\end{array}$ & $\begin{array}{l}0.97 x+6 \\
\text { CC: } 0.98 \\
\text { MB: }-0.2^{\circ} \\
\text { rms : } 15^{\circ}\end{array}$ \\
\hline $\begin{array}{l}\text { SWIM partition } \\
\text { matched to } \\
\text { MFWAM } \\
\text { wind-wave }\end{array}$ & $\begin{array}{l}0.89 \mathrm{x}-0.20 \\
\text { CC: } 0.94 \\
\text { MB: }-0.43 \mathrm{~m} \\
\mathrm{rms}: 0.29 \mathrm{~m} \\
\text { SI: } 17 \%\end{array}$ & $\begin{array}{l}0.87 \mathrm{x}+19 \\
\text { CC: } 0.83 \\
\text { MB: } 9.2 \mathrm{~m} \\
\text { rms : } 23 \mathrm{~m} \\
\text { SI: } 26 \%\end{array}$ & $\begin{array}{l}0.96 x+8.0 \\
\text { CC: } 0.98 \\
\text { MB: }-0.6^{\circ} \\
\text { rms : } 16^{\circ}\end{array}$ \\
\hline
\end{tabular}

Table 8: Statistical scores of the comparisons of wave height, dominant wavelength and dominant direction from SWIM-beam $10^{\circ}$ and MFWAM. Each cell gives the linear regression, the correlation coefficient (CC), the mean bias (MB), the rms difference, the scatter index (SI- except for the direction in this latter case). Number of points is 105024 (data from $26^{\text {th }}$ April to $8^{\text {th }}$ May 2019). See text for other details.

association between partitions in this case. The second one with overestimated wavelengths when MFWAM values are around $100 \mathrm{~m}$. The reason for this cloud of outliers is not very clear yet, but it may be partially due to the presence of some parasitic peaks in the wave spectra (see also section VIII-B) which are not well filtered at the present stage (for the validation phase, no rejection of data based on a criterion on non-homogeneous scenes was applied).

In spite of these difficulties, we can conclude on an overall good assessment for the parameters of the first SWIM partitions: the statistics indicate that $56 \%$ of samples exhibit a difference in wave height smaller than $\pm 30 \mathrm{~cm}, 69 \%$ with a difference less than $\pm 32 \mathrm{~m}$ in wavelength, $78 \%$ with difference in direction less than $\pm 25^{\circ}$. In a second step, the parameters of SWIM and MFWAM partitions were analyzed by imposing a cross-assignment between SWIM and MFWAM partitions. This was carried out, by matching pairs of partitions which minimize the distance in the wavenumber space, as proposed in [26]. This somehow constrains the results on the direction and wavelength but not on the significant wave height.

As expected, the results are quite good for the wavelengths and directions, significantly better than without crossassignment of partitions as indicated by slopes of the regression line which are closer to 1, and smaller rms differences. The best results are for the wind-wave partitions of MFWAM matched to a SWIM partition, with the slope of the regression close to unity and rms differences of $\sim 27 \mathrm{~m}$ for the wavelength and $\sim 16^{\circ}$ for the direction. For the wave heights the main result is that the underestimation of SWIM significant wave height above 1.5 to $2 \mathrm{~m}$ still remains when the partitions are matched. So this confirms the trend found with the full spectra and with the first approach of partition analysis.

We also analyzed the partitions parameters cross-assigned by their wave height values. By doing this, the results on wavelength and direction are not constrained. In this case the results (not illustrated here) are very close to those of the first step (without cross-assignment) with in particular, the same outlier population on wavelength and direction. This confirms that these outliers are mainly due to mis-association between partitions. Part of this mis-association is due to the limit of SWIM detection in the along-track direction, and another part to a different ranking of partitions between SWIM and MFWAM. This test also showed that even with a constraint applied on wave height when associating SWIM and MFWAM partitions, we find an underestimate of SWIM Hs at small wave height and overestimate at large $\mathrm{Hs}$ (regression line $0.78 x+0.24)$.

Finally, in a third step, we compared wave parameters estimated on fully matched partitions. In this case, the partitions were a posteriori calculated on symmetrized MFWAM spectra and overlaid on SWIM spectra. Parameters of the new SWIM partitions were then calculated with same partition contours as in the case of MFWAM spectra. As this approach strongly constrains the wavelengths and directions, we only used this approach in complementary to the other comparisons, in particular to discuss the relative scores between SWIM beams and between partitions. Tables 9, 10, 11 in the Appendix give the results of this analysis. The first comment is that the parameters from beam $10^{\circ}$ give the best agreement with the MFWAM compared to the cases from the other SWIM beams. This is true for all parameters and all statistical indicators (mean bias, rms difference, scatter index). The reason is probably twofold: the beam $10^{\circ}$ is the less sensitive to possible wind fluctuations within the footprint, and it is associated with the smallest speckle noise perturbation. The mean bias on $\mathrm{Hs}$ has as similar order of magnitude for the 3 beams $(0.21 \mathrm{~m}$ to $0.31 \mathrm{~m}$ when considering all the partitions). This results from a small positive bias of SWIM with respect to MFWAM for $\mathrm{Hs} \sim<3 \mathrm{~m}$ and negative bias at larger Hs. For beam $10^{\circ}$ for example, the mean relative bias is between $14 \%$ and $2 \%$ for Hs between 1.5 and $3 \mathrm{~m}$ but is only $4 \%$ for Hs of $6 \mathrm{~m}$. Almost similar biases values are obtained for the other beams, indicating that the speckle correction compensates adequately the differences in the noise levels evidenced in the data (Section VII). The rms difference on $\mathrm{Hs}$ is the smallest for beam $10^{\circ}(0.41 \mathrm{~m}$ when considering all partitions) and the largest for beam $6^{\circ}(0.58 \mathrm{~m}$ when considering all partitions). For a given SWIM beam it decreases with the rank of the partition, but this is probably due to the range of $\mathrm{Hs}$ values encountered for each partition ([0$10] \mathrm{m},[0-6] \mathrm{m}$, and $[0-4 \mathrm{~m}]$ for the $1^{\text {st }}, 2^{\text {nd }}$ and $3^{\text {rd }}$ partition, respectively). As for the direction, the mean bias is less than $1^{\circ}$ (a good correspondence was expected because the partitions are forced to the same contours), while the rms difference is between $19^{\circ}$ (beam $10^{\circ}$ considering all partitions) and $26^{\circ}$ (beam $6^{\circ}$, considering all partitions). This is slightly more, although still comparable, at least for beam $10^{\circ}$, with the initial goal which was specified to $15^{\circ}$. Finally, for the wavelength of the partitions, the rms difference is between $58 \mathrm{~m}$ (beam $10^{\circ}$, $1^{\text {st }}$ partition) and $76 \mathrm{~m}$ (beam $6^{\circ}, 2^{\text {nd }}$ partition). The scatter plots (not shown) indicate that this relatively large rms difference is 
due to some outliers, with SWIM wavelengths spanning over the 200-500 m range while MFWAM values are less than about $200 \mathrm{~m}$. This is similar to what was illustrated in Fig.19c and named "second region of outliers". The scatter plot also confirms that the other region of outliers identified in Fig.19c does not exist when the partitions are imposed with the same contours as in MFWAM. This confirms our comment on Fig.19c in which we attributed these outliers to mis-association between partitions.

Overall all the results obtained with our statistical analysis (several approaches) indicate that the best agreements of SWIM parameters with respect to MFWAM are found for the $10^{\circ}$ beam. They also show that a very good estimation of the direction is obtained when errors in cross-assignment are minimized (almost no biases, $\mathrm{rms} 15^{\circ}-20^{\circ}$ for the beam $10^{\circ}$ ) but the quality is degraded for waves propagating close to the along-track direction in spite of the proposed ad'hoc treatment of speckle noise. Similarly, biases on wavelengths are generally small, and the rms are of the order of magnitude of $30 \mathrm{~m}$ to 75 $\mathrm{m}$ depending on the approach used. The limitations in the detection of waves propagating close to the along-track direction and/or the presence of parasitic peaks in the SWIM spectra may explain a large part of these rms values on wavelengths.

\section{B. Three case studies}

In this section we present three specific studies. The first two illustrate comparisons between wave spectra or wave parameters from SWIM and from in situ measurements (complemented by MFWAM model and/or SAR data).

The third one corresponds to an analysis of wave evolution in a fetch limited case.

\section{1) Comparison of SWIM, buoy and model wave spectra}

In this case buoy measurements are compared to SWIM spectra converted into wave height spectra as a function of frequency, taking into account the dispersion relation in deep water. We discuss here two situations, the first one (case 1) corresponding to a young swell system and a second one (case 2) to a mixed sea condition.

For case 1 the comparison is carried out at the location of the Brittany buoy data $\left(47^{\circ} 33^{\prime} 0^{\prime \prime} \mathrm{N} 8^{\circ} 28^{\prime} 12^{\prime \prime}\right.$ on August $19^{\text {th }} 2019$, 18:00 UTC). This case corresponds to a young swell with Hs $\sim 2 \mathrm{~m}$ from NW generated in the Atlantic open ocean. Figure 21 compares the $1 \mathrm{D}$ and $2 \mathrm{D}$ spectra from the Brittany buoy, the SWIM data, and the MFWAM model. The peak and shape of the 1D spectrum from SWIM are in good agreement with the buoy and the model. However, SWIM data show two shortcomings: the energy is underestimated in the swell energy part, and there is a parasitic peak at low frequency. The underestimation of energy or wave height is compatible with the statistical analysis discussed in VIII-2. The parasitic peak (corresponding to wavelengths close to the $500 \mathrm{~m}$ wavelength limit) is probably due to one of these two factors: either lack of homogeneity over the SWIM footprint, which induces energy at low wave number, or remaining presence of noise floor of the slope spectra which is significantly amplified at these (a)

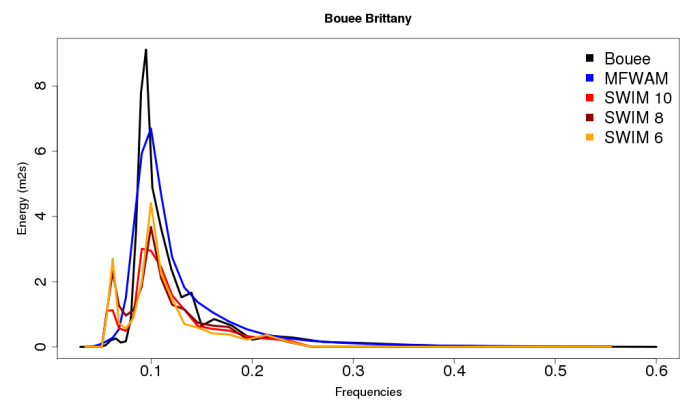

(b)

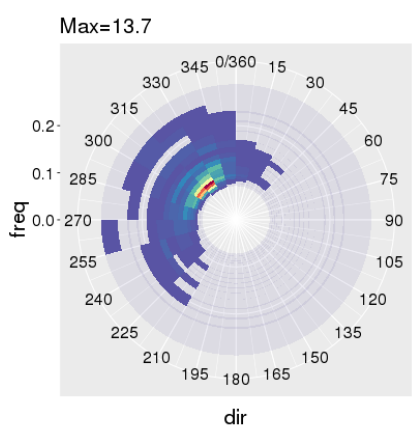

(c)

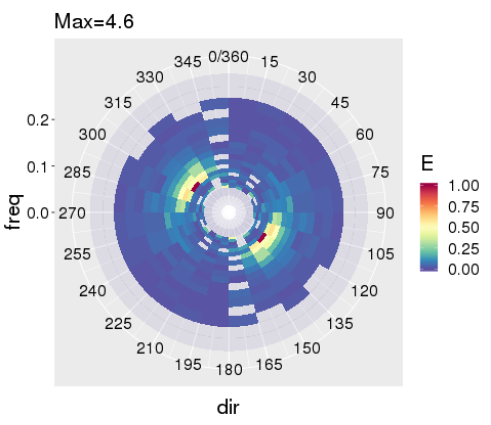

Figure 21(a) Omni-direction spectra $\left(\mathrm{m}^{2} / \mathrm{s}\right.$ ) from the Brittany buoy

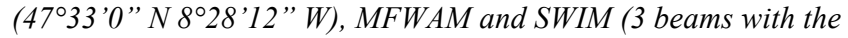
red, brown and yellow curve as indicated in the insert) on August $19^{\text {th }}$ 2019 18:00 UTC. (b, c) directional spectrum from (b) the buoy, (c) SWIM beam $10^{\circ}$. In $(b, c)$, the color scale is relative to the maximum of energy of each plot.

wavelengths when converting to wave height spectra. These two perturbations will be studied in more details in the future, and improvements are expected by applying additional quality controls, in particular to identify and reject non homogenous scenes (work under progress). Note that on the other hand the good consistency in the shape of the spectra with frequency is an indirect indication that the linear MTF is appropriate.

The second case corresponds to a mixed sea condition generated by a mid-latitude storm in the Atlantic waters. According to the buoy observations, there is first a wind sea from South with a $0.2 \mathrm{~Hz}$ peak frequency. As shown in Figure 22, the main part of this component is captured by SWIM, although its energy seems underestimated. The correspondence with respect to the buoy is much better for SWIM spectra obtained with the beams at $8^{\circ}$ and $10^{\circ}$ incidence than with the 
beam at $6^{\circ}$. There are also two swell systems, one around 0.16 $\mathrm{Hz}$ and one around $0.09 \mathrm{~Hz}$. Both swell systems are well identified by SWIM which better captures the second swell from West than the model. As observed for the previous case, there is also an important parasitic peak at low frequency compared to the buoy and MFWAM spectra.

(a)

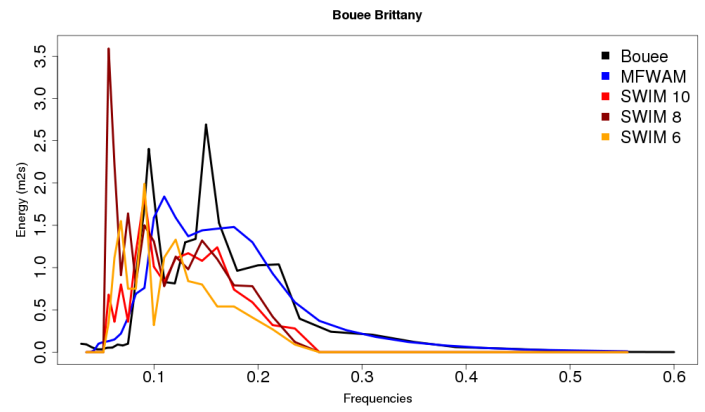

(b)

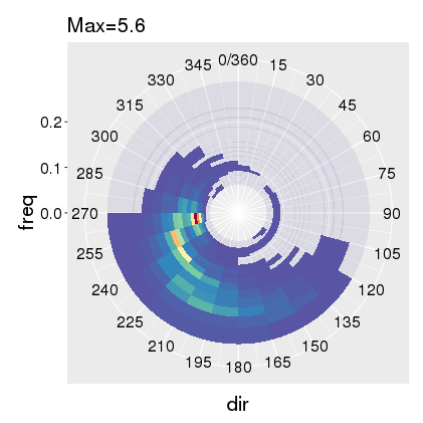

(c)

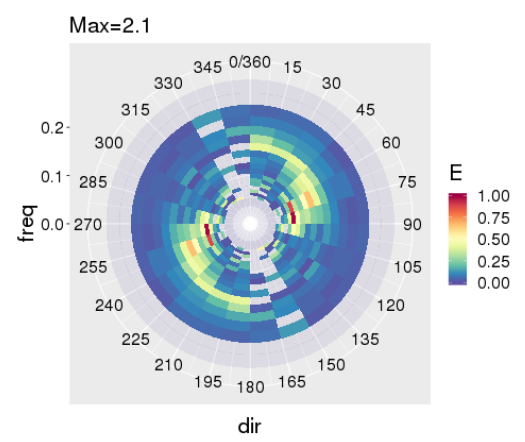

Figure 22: Same as Fig. 21 but for a case on July $24^{\text {th }}$ 2019,18:00UTC

\section{2) Time series of wave parameters in the Pacific Ocean}

The second illustration deals with comparisons of SWIM data with in situ measurements located close to the island of Moorea in the South Pacific $\left(-149.82^{\circ} \mathrm{E},-17.61^{\circ} \mathrm{N}\right)$. The in situ wave parameters were obtained by analyzing $1 \mathrm{~Hz}$ time series of a pressure probe (OSSI probe) installed on the outer slope of the "Paroa" coral reef. CFOSAT data at a distance ranging from 25 to $300 \mathrm{~km}$ from the in situ probe are considered in this analysis. A comparison between SWIM data and in situ data is reported in Fig. 23. It confirms that the local variations of Hs with time are well detected by SWIM. It is however fair to note that Hs values estimated from the off-nadir beams appear systematically slightly overestimated (Fig. 23a). As for the dominant wavelength (Fig. 23b), there is a good agreement for all SWIM beams. SWIM captures very well the dominant wavelength variations with time, with changes from 100 to 500 $\mathrm{m}$ or vice versa observed over short time periods (of the order of a day).
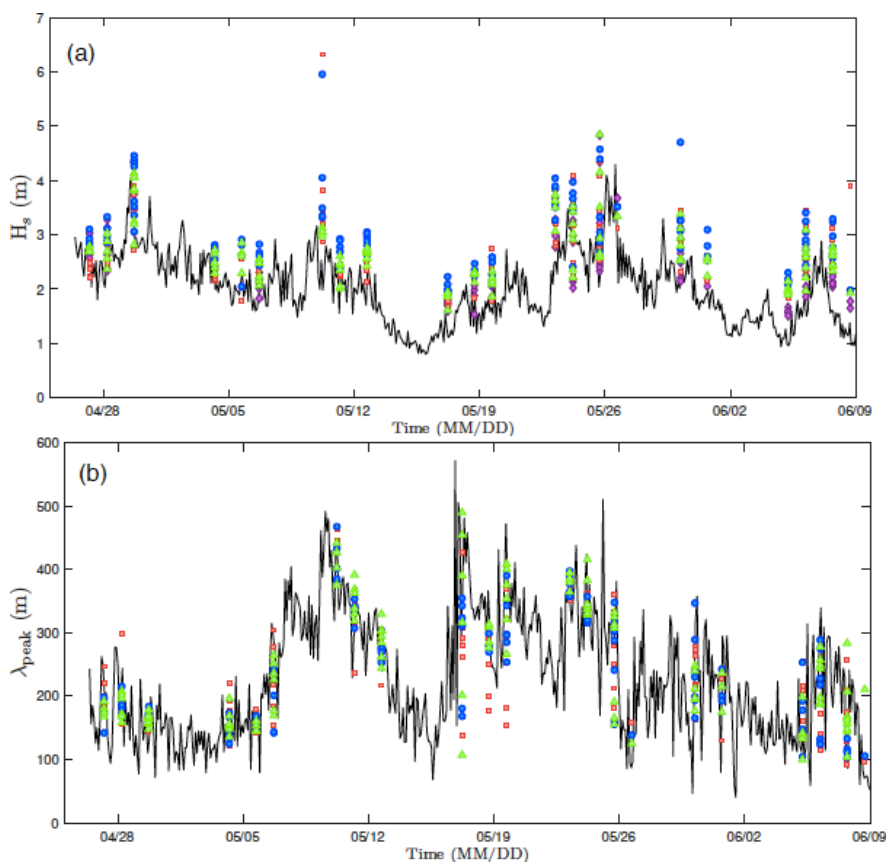

Figure 23: Time series of (a) Hs in $m$ (b) peak wavelength in $m$ from SWIM and from the OSSI probe in Moorea $\left(-149.82^{\circ} \mathrm{E},-17.61^{\circ} \mathrm{N}\right)$ between April $28^{\text {th }} 2019$ and June $9^{\text {th }} 2019$. The black curve indicates the in situ measurements. The CFOSAT nadir measurements are reported in magenta in panel a. The CFOSAT 6,8 and $10^{\circ}$ beams are reported in red, blue and green (panels $a$ and $b$ ).

\section{3) Interest of SWIM observations for studies of wave evolution at regional case}

The advantage of the multi-angular and continuous sampling of the SWIM geometry of observation is illustrated for a situation of waves evolving under fetch limited conditions. These fetch-limited conditions, are frequent in the North Mediterranean Sea under "Tramontane" or "Mistral" wind events which blow from the coast with a constant direction and almost constant wind speed from North or North-West. Such an event occurred during a CFOSAT passage, on April $29^{\text {th }} 2019$ with a wind from Northwest and a wind speed of about 11 to 14 $\mathrm{m} / \mathrm{s}$. The orientation of the satellite track with respect to the coast, and the sampling at different incidence angles with rotating geometry, allows to estimate wave spectra for multiple fetch distances with SWIM observations from the beams $4^{\circ}, 6^{\circ}$, $8^{\circ}$, and $10^{\circ}$. For this specific study, the SWIM directional spectra were evaluated directly from the fluctuation spectra of the $\mathrm{L} 1 \mathrm{~b}$ product (no resampling in the wave box of L2 products), to analyze the evolution of the spectral peak wavenumber with the fetch distance. The results are shown in Fig 24a where they are complemented by the same analysis 
performed on Sentinel-1b images (SAR in image mode) and compared to the WW3 model results. It shows that in this condition of almost constant wind along the fetch direction, and at fetches between 80 and $250 \mathrm{~km}$, the peak wavenumber follows the expected Jonswap law [29] accounting for a wind speed of $11.5 \mathrm{~m} / \mathrm{s}$. After $250 \mathrm{~km}$ the peak wavenumber stabilizes, reaching conditions of to fully-developed wave conditions. It is remarkable to note that with the SWIM data it is possible to follow continuously the increase of the dominant wavelength from about $80 \mathrm{~m}$ to $160 \mathrm{~m}$ over $170 \mathrm{~km}$ of fetch distance. The WW3 model results also follow this law but the peak wavenumbers are biased high with respect to the observations. This probably indicates some bias in the wind or stress forcing of WW3. This analysis of the presented dataset illustrates the interest of SWIM for characterizing the spatial structure of a wave field at a regional scale. This is essential for wave model improvement and calibration.
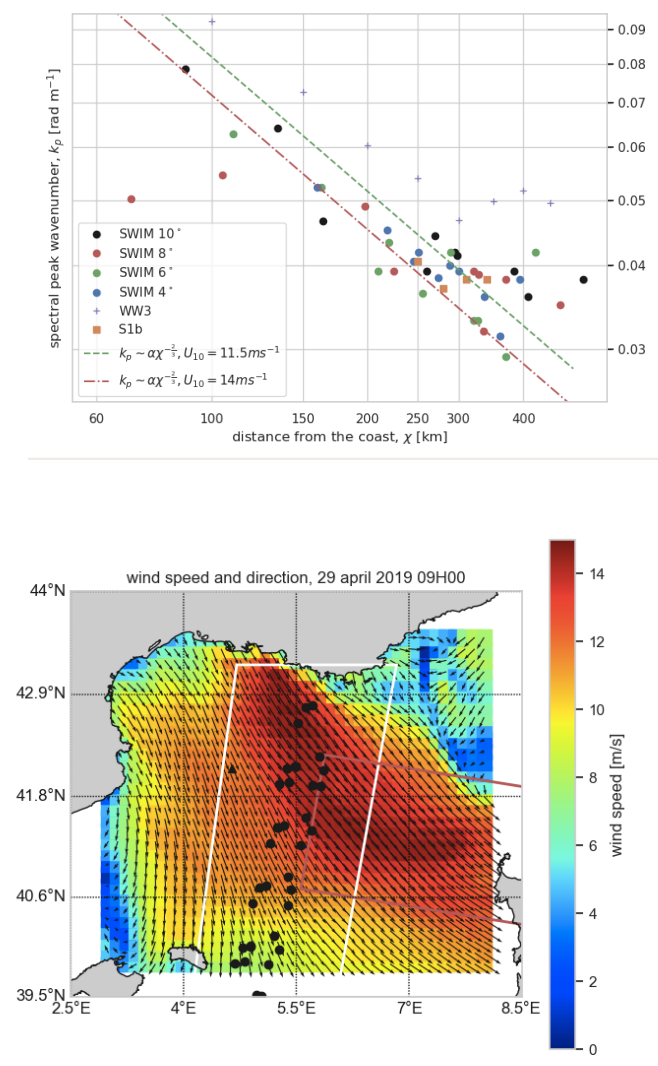

Figure 24: Case of April $29^{\text {th }} 2019$ in the North Mediterranean sea. (a) Peak frequency as a function of the fetch (ie distance to the coast along the wind direction-see Fig. 24 b), estimated from SWIM (color dots for SWIM beams $4^{\circ}, 6^{\circ}, 8^{\circ}$, and $10^{\circ}$ ), from S-1B SAR image (square orange symbols) and from $W W 3$ (plus signs). The two lines represent a fit of the wavenumber following a power law as a function of the distance of fetch using the SWIM and S-1B for a constant wind speed of $11.5 \mathrm{~m} / \mathrm{s}$ (green dashed line) and $14 \mathrm{~m} / \mathrm{s}$ (red dashed-dotted line). (b) Wind vector field and position of data sets. The SWIM $10^{\circ}$ beam swath extension is shown with a white frame, black dots represent coordinates of SWIM data used in Fig.24a, Sentinel-1b image position is shown with a red frame.

\section{IX- SYNTHESIS AND CONCLUSION}

In this paper we have presented the first analysis of the geophysical products provided by the SWIM instrument onboard the CFOSAT satellite.

We have first illustrated the good instrumental behavior of the instrument. Then, we have illustrated the high quality of data obtained from the nadir observations (significant wave height, normalized radar cross-section and wind speed). Compared to the Jason 3 products, the performance on these parameters is of the same order in spite of a lower acquisition rate, but thanks to the new "adaptive" retracking algorithm. We have shown the consistency of the normalized radar cross-sections $\sigma_{0}$ for all incidence beams (except for the $2^{\circ}$ beam which still needs to be assessed). Without any a posteriori calibration, the trend of $\sigma_{0}$ with incidence and with wind speed is very similar to that provided by GPM data, and the bias with respect to mean GPM values is less or of the order of $1 \mathrm{~dB}$.

The level $1 \mathrm{~b}$ data include fluctuation of radar cross-section which are supposed to be maximum when the radar look direction is aligned with the wave propagation. The density spectra of these fluctuations have been compared to wave spectra from the WW3 model. We have found a good correlation for all wavenumbers of interest $\left(1.25 \times 10^{-2}\right.$ to 8.98 $\left.\mathrm{x} 10^{-2} \mathrm{rad} \mathrm{m}^{-1}\right)$ except in a sector of about $\pm 15^{\circ}$ around the direction of the satellite track. The best correlation is obtained for SWIM beams at $8^{\circ}$ and $10^{\circ}$ incidences. The decrease of correlation in the azimuthal sector aligned with the satellitetrack is attributed to the increase of noise due to the decrease of the radar Doppler Bandwidth in this direction. An empirical model was built by analyzing the SWIM data themselves to parameterize the density spectrum of speckle both within and outside this perturbed angular. It was shown that within the angular sector affected by the decrease of radar Doppler bandwidth, the speckle intensity varies with latitude and with sea surface conditions. In opposite, for all other directions, for which the Doppler Bandwidth is larger than the Pulse Repetition Frequency, the speckle noise level is governed by the number of samples averaged in time and in radial distance.

Finally, the evaluation of the wave spectra and their main parameters (wave height dominant direction and dominant wavelength) was presented. The analysis performed by using the correlation index proposed by [26] allows to conclude that the best (resp. lowest) consistency with model spectra are obtained for the $10^{\circ}$ beam (resp. $6^{\circ}$ beam) observations.

The quality of the wave parameters was assessed using two approaches: comparison of wave parameters from SWIM and the MFWAM model spectra without partitioning, and comparison of parameters of SWIM and MFWAM spectral partitions. In this latter case, in order to reach conclusions not biased by the difference in partitions, we discussed three kind of comparisons: i) parameters from separate partition estimations (without cross-assignment), ii) parameters from SWIM partitions matched to MFWAM partitions obtained by minimizing the dominant wavenumber vector distance or the distance in wave height, iii) parameters obtained by overlaying MFWAM partitions on the SWIM spectra. Overall the results indicate that:

i) SWIM observations from beam $10^{\circ}$ give the best results, while the beam $6^{\circ}$ gives the less satisfactory results. This may 
be explained by the smallest sensitivity of $\sigma_{0}$ to wind speed close to the $10^{\circ}$ incidence and by the smallest contribution of speckle contribution at this incidence (largest number of averaged echoes

ii) the significant wave height is overestimated at wave heights smaller than 2 to $3 \mathrm{~m}$ (depending on the method used for the comparison), and underestimated at larger wave heights. This shortcoming, probably due to remaining uncertainties in the noise correction and in the MTF estimation, may be minimized in the future by applying an empirical correction on significant wave heights or by normalizing the spectra with the nadir observation as reference. As for the dispersion, the comparisons with model data indicate a rms difference of $0.26 \mathrm{~m}$ to $0.71 \mathrm{~m}$ depending of the SWIM beam and method used for the evaluation. The rms difference is significantly less for the beam $10^{\circ}$ compared to the other beams and for the full spectrum and first partition, indicating that when a good accuracy is required on wave height, it is preferable to focus on the $10^{\circ}$ beam full spectrum or $1^{\text {st }}$ partition data.

iii) the direction of the waves is generally well retrieved except in some of the conditions where waves propagate in the along-track sector (there are larger limitations for long swell cases in this case). The best consistency with model and in situ data are for the beam $10^{\circ}$, and the least one with the beam $6^{\circ}$. The rms differences with respect to the same reference (MFWAM model) are of the order of $15^{\circ}$ to $29^{\circ}$ depending of the method of analysis.

iv) the dominant wavelengths, as identified on the wave slope spectra, are also well retrieved except in the along-track directions and mainly for the long swell. Again, the best results are for the beam $10^{\circ}$ and for the $1^{\text {st }}$ SWIM partition. The order of magnitude of the rms differences with respect to the model are typically of $30 \mathrm{~m}$ to $40 \mathrm{~m}$.

First comparisons with in situ observations confirm the main characteristics obtained with the statistical analysis. For some cases, a parasitic peak at low wavenumber is identified in the spectra. It is likely due to an amplification of the noise floor when converting slope spectra to height spectra.

Overall the analysis presented in this paper shows that SWIM is able to provide the spectral properties of ocean waves in the wavelength range $[70 \mathrm{~m}-500 \mathrm{~m}]$. Although no detailed quality control was applied at this stage (like removing scenes affected by rain or non-homogeneous conditions), we showed that SWIM products already bring valuable information on wave spectra details and wave parameters which are very complementary to in situ observations, other satellite observations, or models. In particular, the evolution of spectral properties at the regional scale seems very promising, even in coastal conditions.

Further improvements on the wave products are expected with the planned introduction of quality controls (rejection of nonhomogenous wind or wave scenes, scenes impacted by rain, etc), better filtering of the energy of low wavenumbers, and refinement of the Modulation Transfer Function. In addition, downstream of the inversion, wave partitions as determined from the operational algorithm will be more constrained in order to reject partitions with too low energy, or to merge partitions which are close in direction. Work is under progress to assess these new improvements and will be reported in future papers. Also, more extensive comparisons with in situ data will be carried out, in particular using the NOAA buoy network. This requires however to reprocess SWIM data according to the improvements presented in this paper over a long period of time to allow for a large comparison data set.

\section{ACKNOWLEDGMENTS}

The authors are very grateful to the reviewers for their helpful comments and encouragements. The authors wish to acknowledge the major role of the colleagues who contributed to the success of the mission and instrument performance. In particular, P. Castillan and J.-M. Lachiver as project SWIM managers in the development and operational phase, respectively, N. Corcoral as system engineer, T. Amiot as SWIM instrument responsible. We have a thought for $\mathrm{G}$. Boutonnet, the former project manager of the French ground segment, who left us prematurely. SWIM was built by Thales Alenia Space Industry (TAS) under a CNES contract. Many thanks to L. Rey and B. Carrayon from TAS for their role in the SWIM design, realization and tests. F. Nouguier (LOPS) and F. Collard (Oceandatalab) helped to interpret the fluctuation spectra and speckle behavior thanks to their numerical approaches. Comparisons with in situ data in the Pacific Ocean were carried out thanks to the contribution of F. Zuberer at CRIOBE (France) for the in situ measurements. Thanks also to B. Perret at CLS, C. Dufour at LATMOS who developed the main part of the computer architecture, data bases and software used for the data analysis presented here. All CFOSAT products are provided by courtesy of CNSA and CNES. 
APPENDIX

\begin{tabular}{|c|c|c|c|}
\hline Hs & Beam $6^{\circ}$ & Beam $8^{\circ}$ & Beam $10^{\circ}$ \\
\hline $\begin{array}{l}\mathrm{Nb} \text { of } \\
\text { points }\end{array}$ & $\begin{array}{l}\text { All : } 570498 \\
\text { part } 1: 250130 \\
\text { part } 2: 197381 \\
\text { part } 3: 122987\end{array}$ & $\begin{array}{l}\text { All : } 567891 \\
\text { part } 1: 249050 \\
\text { part } 2: 196427 \\
\text { part } 3: 122414\end{array}$ & $\begin{array}{l}\text { All : } 556333 \\
\text { part } 1: 243736 \\
\text { part 2: } 192482 \\
\text { part } 3: 120115\end{array}$ \\
\hline $\begin{array}{l}\text { Mean Bias } \\
{[\mathrm{m}]}\end{array}$ & $\begin{array}{l}\text { All : } 0.23 \\
\text { part } 1: 0,16 \\
\text { part } 2: 0,28 \\
\text { part } 3: 0,29\end{array}$ & $\begin{array}{l}\text { All : 0,31 } \\
\text { part } 1: 0,30 \\
\text { part } 2: 0,33 \\
\text { part } 3: 0,30\end{array}$ & $\begin{array}{l}\text { All : 0,21 } \\
\text { part } 1: 0,17 \\
\text { part } 2: 0,24 \\
\text { part } 3: 0,24\end{array}$ \\
\hline $\begin{array}{c}\text { RMSE } \\
{[\mathrm{m}]}\end{array}$ & $\begin{array}{l}\text { All : } 0.58 \\
\text { part } 1: 0,71 \\
\text { part } 2: 0,50 \\
\text { part } 3: 0,39\end{array}$ & $\begin{array}{l}\text { All : 0,54 } \\
\text { part } 1: 0,65 \\
\text { part } 2: 0,48 \\
\text { part } 3: 0,35\end{array}$ & $\begin{array}{l}\text { All : } 0,41 \\
\text { part } 1: 0,49 \\
\text { part } 2: 0,34 \\
\text { part } 3: 0,29\end{array}$ \\
\hline $\begin{array}{l}\text { Scatter } \\
\text { Index }\end{array}$ & $\begin{array}{l}\text { All : 40,3 } \\
\text { part } 1: 30,4 \\
\text { part } 2: 51,4 \\
\text { part } 3: 84,8\end{array}$ & $\begin{array}{l}\text { All : } 37,2 \\
\text { part } 1: 28,2 \\
\text { part } 2: 49,3 \\
\text { part } 3: 74,4\end{array}$ & $\begin{array}{l}\text { All : } 27,9 \\
\text { part } 1: 21,2 \\
\text { part } 2: 34,5 \\
\text { part } 3: 62,7\end{array}$ \\
\hline
\end{tabular}

Table 9: Statistical scores for the comparison of the significant wave height of the MF-WAM wave spectra partitions and of the SWIM spectra partitions when the partitions of MFWAM are overlaid on the SWIM spectra. The data set covers from April $25^{\text {th }}$ to June $9^{\text {th }} 2019$

\begin{tabular}{|c|c|c|c|}
\hline Dir & Beam $6^{\circ}$ & Beam $8^{\circ}$ & Beam $10^{\circ}$ \\
\hline $\begin{array}{l}\text { Mean Bias } \\
\text { [deg.] }\end{array}$ & $\begin{array}{l}\text { All : }-0,4 \\
\text { part } 1:-0,8 \\
\text { part } 2: 0,0 \\
\text { part } 3:-0,2\end{array}$ & $\begin{array}{l}\text { All : }-0,5 \\
\text { part } 1:-0,9 \\
\text { part } 2:-0,1 \\
\text { part } 3:-0,2\end{array}$ & $\begin{array}{l}\text { All : }-0,5 \\
\text { part } 1:-0,6 \\
\text { part } 2:-0,3 \\
\text { part } 3:-0,4\end{array}$ \\
\hline RMSE [deg.] & $\begin{array}{l}\text { All }: 26,4 \\
\text { part } 1: 29,0 \\
\text { part } 2: 26,0 \\
\text { part } 3: 21,4\end{array}$ & $\begin{array}{l}\text { All }: 23,4 \\
\text { part } 1: 24,8 \\
\text { part } 2: 23,5 \\
\text { part } 3: 20,3\end{array}$ & $\begin{array}{l}\text { All : } 19,2 \\
\text { part } 1: 19,3 \\
\text { part } 2: 19,5 \\
\text { part } 3: 18,4\end{array}$ \\
\hline
\end{tabular}

Table 10: Same as Table 9 but for the direction of the partitions

\begin{tabular}{|c|c|c|c|}
\hline W1 & Beam $6^{\circ}$ & Beam $8^{\circ}$ & Beam $10^{\circ}$ \\
\hline $\begin{array}{c}\text { Mean Bias } \\
{[\mathrm{m}]}\end{array}$ & $\begin{array}{l}\text { All : 13,9 } \\
\text { part } 1: 11,4 \\
\text { part } 2: 14,4 \\
\text { part } 3: 18,4\end{array}$ & $\begin{array}{l}\text { All }: 8,3 \\
\text { part } 1: 3,9 \\
\text { part } 2: 9,4 \\
\text { part } 3: 15,6\end{array}$ & $\begin{array}{l}\text { All : 5,6 } \\
\text { part } 1: 0,2 \\
\text { part 2:7,0 } \\
\text { part 3: 14,3 }\end{array}$ \\
\hline $\begin{array}{c}\text { RMSE } \\
{[\mathrm{m}]}\end{array}$ & $\begin{array}{l}\text { All : 73,5 } \\
\text { part } 1: 73,8 \\
\text { part } 2: 75,6 \\
\text { part } 3: 69,3\end{array}$ & $\begin{array}{l}\text { All }: 67,3 \\
\text { part } 1: 65,5 \\
\text { part } 2: 70,2 \\
\text { part } 3: 65,3\end{array}$ & $\begin{array}{l}\text { All : } 62 \\
\text { part } 1: 57,9 \\
\text { part } 2: 66,0 \\
\text { part } 3: 62,5\end{array}$ \\
\hline $\begin{array}{l}\text { Scatter } \\
\text { Index }\end{array}$ & $\begin{array}{l}\text { All : 35.6 } \\
\text { part } 1: 39,7 \\
\text { part } 2: 36,9 \\
\text { part } 3: 27,5\end{array}$ & $\begin{array}{l}\text { All }: 32,5 \\
\text { part } 1: 35,2 \\
\text { part } 2: 34,3 \\
\text { part } 3: 25,9\end{array}$ & $\begin{array}{l}\text { All : 30,0 } \\
\text { part } 1: 31,1 \\
\text { part } 2: 32,2 \\
\text { part } 3: 24,8\end{array}$ \\
\hline
\end{tabular}

Table 11: Same as Table 9 but for the wavelength of the partitions

\section{REFERENCES}

[1] Hauser D., et al., "Overview of the CFOSAT mission", Proceedings of IGARSS'2016, Beijing (China), July 2016
[2] Hauser D., C. Tison, T. Amiot, L. Delaye, N. Corcoral et al, "SWIM: the first spaceborne wave scatterometer", IEEE Trans. on Geoscience and Remote Sensing, 10.1109/TGRS.2017.2658672, VOL 55, 5, May 2017

[3] Alpers W. R. and C. Brüning, "On the relative importance of motion related contributions to the SAR imaging mechanism of ocean surface waves," IEEE Trans. Geosci. Remote Sens., vol. GE-24, no. 6, pp. 873885, Nov. 1986.

[4] Li H., B. Chapron, A. Mouche, and J. Stopa, "A new SAR cross-spectral parameter: definition and directional property using the global Sentinel-1 measurements", J. Geopgys. Res. Oceans, 124, 1566-1577, doi: 10.1029/2018JC014638

[5] Dong Xiaolong, Zhu Di, W. Lin, H. Liu, J. Jiang, "A Ku-band rotating fan-beam scatterometer: design and performance simulations", Proceedings of IGARSS'10, 2010

[6] Jackson F. C., W. T. Walton, and P. L. Baker, "Aircraft and satellite measurement of ocean wave directional spectra using scanning-beam microwave radars", J. Geophys. Res., Vol. 90, 987-1004, 1985

[7] Jackson F. C., W. T. Walton, and C. Y. Peng, "A comparison of in situ and airborne radar observations of ocean wave directionality", $J$. Geophys. Res., Vol. 90, 1005-1018, 1985

[8] Hauser D., G. Caudal, G. J. Rijckenberg, D. Vidal-Madjar, G. Laurent, and P. Lancelin, "RESSAC : A new airborne FM/CW radar ocean wave spectrometer", IEEE Transactions on Geoscience and Remote Sensing, 30(5), 981-995, 1992

[9] Caudal G., D. Hauser, R. Valentin, C. Le Gac, "KUROS : A new airborne $\mathrm{Ku}$-band Doppler radar for observation of surfaces", Jour. Atmos. and Oceanic Technology, Vol. 31, No. 10. , 2223-2245, 2014

[10] Jackson, F.C., "Directional spectra from the radar ocean wave spectrometer during LEWEX. In: Directional Ocean Wave Spectra, $R$. Beal (Ed.)", Johns Hopkins University Press, pp. 91—97., 1991

[11] Vandemark, D., F.C. Jackson, E.J. Walsh and B. Chapron, "Airborne radar measurements of ocean wave spectra and wind speed during the grand banks ERS-1 SAR wave experiment", Atmosphere-Ocean, 32:1, 143-178, DOI: 10.1080/07055900.1994.9649493, 1994

[12] Legresy B., Papa F., Remy F., Vinay G., van den Bosch M., Zanife O. Z., "ENVISAT radar altimeter measurements over continental surfaces and ice caps using the ICE-2 retracking algorithm". Remote Sensing of Environment, 95 (2), 150-163. ISSN 0034-4257, 2005

[13] Ardhuin F, Rogers E, Babanin A V, et al, "Semi empirical Dissipation Source Functions for Ocean Waves. Part I: Definition, Calibration, and Validation”, J. Phys. Oceanogr., 40:1917-1941. doi: 10.1175/2010JPO4324.1, 2010

[14] Aouf L., "CMEMS quality information document for global ocean waves analysis and forecasting product", CMEMS-GLO-QUID-001 027 http://resources.marine.copernicus.eu/documents/QUID/CMEMS-GL̄OQUID-001-027.pdf, 2018

[15] Gressani V., D. Nouguier and A. Mouche, "Wave Spectrometer Tilt Modulation Transfer Function Using Near-Nadir Ku-and Ka-Band GPM Radar Measurements", Proceedings of the 2018 IEEE International Geoscience and Remote Sensing Symposium, Valencia (Spain), 2018

[16] Brown, G . S., "The average impulse response of a rough surface and its applications", IEEE Trans. Antenna Propagat. 2, 5(1), 67-74, 1977.

[17] Tourain C., F. Piras, A. Olivier, P. Thibaut, F. Boy, JC Poisson, L. Hermozo, D. Hauser, C. Tison, "CFOSAT SWIM adaptive retracking: description and validation", IEEE Trans on Geoscience anr Remote Sensing, submitted

[18] J. A. Nelder, R. Mead, "A simplex method for function optimization", The Computer Journal, Volume 7, Issue 4, p 308-313, doi 10.1093.7.4.308, 1965

[19] Gourrion, J., D. Vandemark, S. Bailey, B. Chapron, G. P. Gommenginger, P. G. Challenor, and M. A. Srokosz, "A two-parameter wind speed algorithm for Ku-band altimeters", J. Atmos. Oceanic Technol., 19, 20302048, doi:10.1175/1520-0426, 2002

[20] Collard, F., "Algorithmes de vent et période moyenne des vagues JASON à base de réseaux de neurones." BO-021-CLS-0407-RF, Boost Technologies, 33 pp., 2005

[21] J. Tournadre, J. C. Poisson, N. Steunou \& B. Picard, "Validation of AltiKa Matching Pursuit Rain Flag", Marine Geodesy, 38-1, p 107-123, 2015, doi 10.1080/01490419.2014.1001048

[22] Ulaby F. D. Long, W. Blackwell, C. Elachi, A.K. Fung, C. Rif K. Sarabandi,, H. Zebker and J. Van Zyl, "Microwave Radar and Radiometric Remote Sensing", Univ. of Michigan Press, ISBN: 978-0472-11935-6, 2014

[23] Gower, J., "Layover in satellite radar images of ocean waves". $J$. Geophys. Res. Ocean., 88, 7719-7720, 1983. 
[24] Boisot O., L. Amarouche; J.-C. Lalaurie, C.-A. Guérin, "Dynamical properties of sea-surface microwave backscatter at low-incidence: correlation time and Doppler shift", IEEE Transactions on Geoscience and Remote Sensing, 54 (12), 10.1109/TGRS.2016.2601242, 2016

[25] Hanson J.-L.and O. M. Phillips, "Automated analysis of ocean surface directional wave spectra", J. Atmos. Ocean. Technol., vol. 18, no. 2, $277-$ 293, 2001.

[26] Hasselmann S., C. Brüning, K. Hasselmann and P. Heimbach, "An improved algorithm for the retrieval of ocean wave spectra from synthetic aperture radar image spectra", Journal of Geophys. Res. Oceans, Vol 101, N07, p16615-16629, 1996

[27] Aouf L., A. Dalphinet, D. Hauser, L. Delaye, C. Tison; B. Chapron, L. Hermozo, C. Tourain, "On The assimilation of CFOSAT wave data in the wave model MFWAM : Verification phase", Proceedings of the 2019 IEEE International Geoscience and Remote Sensing Symposium, Yokohama (Japan), 2019

[28] Collard, Collard F., F. Ardhuin and B. Chapron, "Monitoring and analysis of ocean swell fields from space: New methods for routine observation", J. Geophys. Res., 114, C07023, doi:10.1029/2008JC005215, 2009

[29] Hasselmann K., T P. Barnett, E Bouws, H. Carlson, D.E. Cartwright, K. Enke, J. A. Ewing, H. Gienapp, D. E. Hasselmann, P. Kruseman, A. Meerburg, P. Muller, D. Olbers, K. Richter, W. Sell, and H. Walden, "Measurements of wind-wave growth and swell decay during the joint North Sea wave, project (Jonswap)", Deut. Hydrogr. Z. , 8 :1-95, 011973 